\title{
Impact of lipid rafts on the $T$-cell-receptor and peptide-major-histocompatibility-complex interactions under different measurement conditions
}

\author{
Long $\mathrm{Li},{ }^{1}$ Guang-Kui Xu, ${ }^{2}$ and Fan Song ${ }^{1,3, *}$ \\ ${ }^{1}$ State Key Laboratory of Nonlinear Mechanics (LNM) and Beijing Key Laboratory of Engineered Construction and Mechanobiology, \\ Institute of Mechanics, Chinese Academy of Sciences, Beijing, 100190, China \\ ${ }^{2}$ International Center for Applied Mechanics, State Key Laboratory for Strength and Vibration of Mechanical Structures, \\ School of Aerospace, Xi'an Jiaotong University, Xi'an, 710049, China \\ ${ }^{3}$ School of Engineering Science, University of Chinese Academy of Sciences, Beijing 100049, China \\ (Received 2 July 2016; revised manuscript received 3 November 2016; published 10 January 2017)
}

\begin{abstract}
The interactions between T-cell receptor (TCR) and peptide-major-histocompatibility complex (pMHC), which enable T-cell development and initiate adaptive immune responses, have been intensively studied. However, a central issue of how lipid rafts affect the TCR-pMHC interactions remains unclear. Here, by using a statisticalmechanical membrane model, we show that the binding affinity of TCR and pMHC anchored on two apposing cell membranes is significantly enhanced because of the lipid raft-induced signaling protein aggregation. This finding may provide an alternative insight into the mechanism of T-cell activation triggered by very low densities of pMHC. In the case of cell-substrate adhesion, our results indicate that the loss of lateral mobility of the proteins on the solid substrate leads to the inhibitory effect of lipid rafts on TCR-pMHC interactions. Our findings help to understand why different experimental methods for measuring the impact of lipid rafts on the receptor-ligand interactions have led to contradictory conclusions.
\end{abstract}

DOI: 10.1103/PhysRevE.95.012403

\section{INTRODUCTION}

The prominent features in the adaptive immune response involve the activation of mature $\mathrm{T}$ lymphocytes ( $\mathrm{T}$ cells) and the selection and maturation of immature T cells [1]. These critical events are all initiated by the interactions of the T-cell receptor (TCR) on the surface of $\mathrm{T}$ cells with a particular ligand, peptide-major-histocompatibility complex (pMHC) on the surface of antigen presenting cells (APCs) [2,3]. The recognition of pMHC by TCR then leads to the intracellular signal transduction events that are required for $\mathrm{T}$ cells development and effector functions. The earliest signaling event that occurs after TCR ligation by pMHC is the phosphorylation of immunoreceptor tyrosine-based activation motifs (ITAMs) in the CD3 subunits of TCR by the Src family tyrosine kinases, Lck and Fyn. This phosphorylation results in the recruitment of the Syk family kinase ZAP-70 to the TCR/CD3 complex where it is activated. Activated ZAP-70 subsequently phosphorylates the transmembrane adaptor linker for activation of $\mathrm{T}$ cells (LAT), which in turn promotes the recruitment and activation of numerous downstream adaptors or scaffold proteins to favor TCR signaling cascades [4-8]. Analogously, upon the binding of TCR to pMHC, thymocytes will undergo either positive or negative selection with similar signaling pathways and associated components involved in mature T-cell activation. Positive selection selects thymocytes with TCR capable of binding pMHC, whereas negative selection induces the apoptosis in thymocytes expressing TCR with high affinity for pMHC $[9,10]$.

Given the significance and complexity of TCR signaling transduction, a large number of studies have been conducted to understand the mechanisms of each step in signaling cascades $[5,8,11,12]$. Recently, the implications

*songf@lnm.imech.ac.cn of lipid rafts in T-cell activation and thymocyte selection have been investigated [4,13-23]. Lipid rafts, cholesteroland sphingolipid-enriched membrane microdomains, exist as distinct liquid-ordered phases that float freely as stable entities in the liquid-disordered matrix of the plasma membrane. They are also resistant to extraction with nonionic detergents. A central feature of lipid rafts is that of selectively recruiting classes of proteins [4,24,25]. Evidence from experiments indicates that many T-cell signaling proteins, including but not limited to TCR, CD4, CD8, Lck, LAT, Ras, Fyn, and ZAP-70, are present in lipid rafts [13]. It is widely accepted that lipid rafts serving as signaling platforms can facilitate protein-protein interactions on a single membrane by virtue of spatial proximity of participating components, thereby promoting signaling transduction $[4,26]$. However, the role of lipid rafts in the primordial step of the TCR signal transduction, TCR-pMHC ligation, has rarely been taken into consideration (Fig. 1). By performing an in situ experiment, Anderson et al. demonstrated that MHC class II molecules constitutively reside in lipid rafts. They showed that disruption of lipid rafts on APCs by the treatment of cells with raft-disrupting-agent, methyl- $\beta$-cyclodextrin $(\mathrm{M} \beta \mathrm{CD})$, inhibits the MHC class II-restricted antigen presentation [27]. They presumed that the increased local concentration of MHC class II molecules in lipid rafts is crucial for the initiation of immune responses, which provides important insights, whereas experimental data of cell-mimetic systems obtained by Murai et al. conversely suggest that lipid rafts exert negative control on the receptor-ligand interactions [28], and the corresponding regulatory mechanism is not yet resolved. Taken together, the fundamental mechanism of how lipid rafts might drive TCR-pMHC interactions and the reason for the different experimental results remain unclear and quantitative analysis is lacking.

In the present study, we will focus on the functional role of lipid raft characteristics in the TCR-pMHC interactions with 


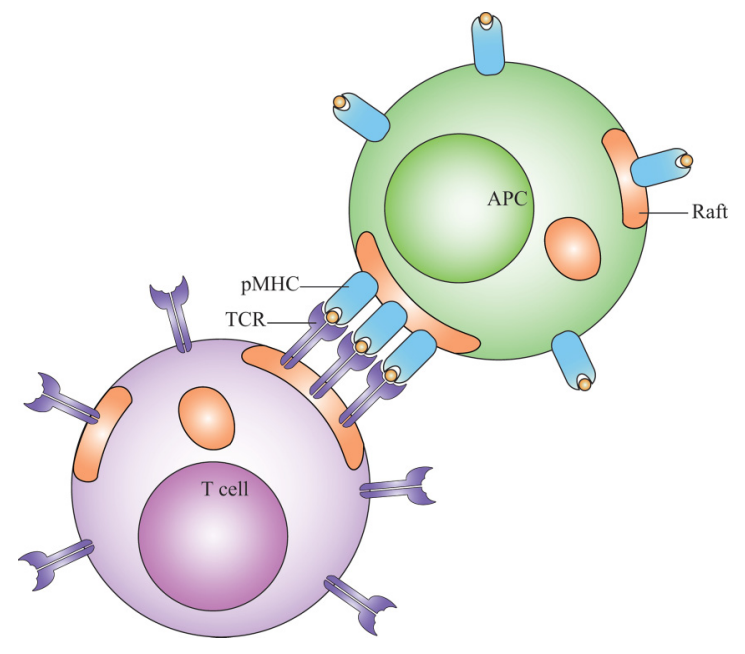

FIG. 1. Illustration of antigen recognition via TCR-pMHC interactions in the presence of lipid rafts.

a recently developed statistical-mechanical membrane model. We find that, consistent with the experimental results [27], the antigen presentation is facilitated by the protein aggregation induced by lipid raft characteristics. Moreover, based on the quantitative analysis that the presence of the lipid rafts leads to a significant increase in the two-dimensional (2D) binding affinity, we provide an alternative insight into the mechanism by which $\mathrm{T}$ cells can be efficiently activated by $\mathrm{pMHC}$ at very low densities. Importantly, we show that lipid rafts can either positively or negatively regulate the receptor-ligand interactions depending on the experimental methods, which helps to reconcile the seemingly discrepant experimental results.

\section{MODEL AND METHOD}

In this study, we use the Monte Carlo (MC) simulation method to investigate the effects of lipid rafts on the TCR-pMHC interactions. The simulation process involves the fluctuation of membranes, and the lateral diffusion of signaling proteins and lipid rafts on the membranes. In the MC simulations, the cell membrane is represented by a discretized elastic sheet consisting of $N \times N$ quadratic lattices of size $a^{2}$. Each lattice can be only occupied by a TCR or a pMHC molecule, which undergoes undirected diffusion described by the hopping process. Whether a binding event of TCR and $\mathrm{pMHC}$ occurs or not depends on the distribution of signaling proteins and the local separation of binding sites. The entropic repulsive force, induced by thermal fluctuations of membrane shapes [29], and specific attraction force, mediated by TCRs and pMHCs, determine the local separations and configurations of the membranes. Here, we use the square-well potential to model the TCR-pMHC interactions. The lipid rafts are modeled as two-dimensional patches with low diffusivity. Here, as studied by Nicolau et al. [30,31], we introduce a ratio $\rho=D_{\text {raft }} / D_{\text {nonraft }}$ of diffusion coefficient of the signaling proteins inside $\left(D_{\text {raft }}\right)$ and outside $\left(D_{\text {nonraft }}\right)$ lipid rafts to describe the extent of the slowdown diffusion of signaling proteins within lipid rafts. Lipid rafts are assumed to diffuse in a manner analogous to signaling proteins. To satisfy the condition of detailed balance, the microdomains in the T-cell membrane and the APC membrane move independently of TCRs and pMHCs, respectively. In this work, we employ two types of raft models, static and dynamic, to study their roles in the TCR-pMHC interactions. In the static model, lipid rafts are represented as square-shaped patches with defined sizes and corresponding diffusion rates. Both shape and size of lipid rafts are fixed throughout a simulation run. To describe the dynamic behavior of lipid rafts in vivo, the neighboring elementary units of lipid rafts experience, in addition to the hard-square repulsion interaction, an attractive cis interaction $U_{\alpha \alpha}$ in the dynamic raft model, where the notation $\alpha$ denotes the raft membrane patches. In the MC simulations, we investigate the impact of dynamic lipid rafts on the TCR-pMHC interactions by varying the value of $c i$ interaction $U_{\alpha \alpha}$.

In view of the fact that proteins can be included or excluded from lipid rafts to variable extents, we introduce a parameter $\Delta U_{\text {affinity }}$ to define the change in the energy for a signaling protein entering into a lipid raft from nonraft regions. When a signaling protein attempts to move from nonraft to raft patches, this movement is accepted or rejected according to the Metropolis criterion. Similarly, the acceptance probability of a raft diffusion attempt depends on the raft affinity for signaling proteins and the change of the number of proteins in this microdomain. In particular, once hopping into a raft (or nonraft) region, a signaling protein will be permanently captured in that region for $\Delta U_{\text {affinity }} \rightarrow-\infty$ (or $\left.\Delta U_{\text {affinity }} \rightarrow+\infty\right)$ through its hopping process. Additionally, we incorporate the interaction potential terms $W_{\text {mem }}, W_{\text {pro }}$, and $W_{\text {raft }}$ into the Hamiltonian of the system to prevent the overlaps of two apposing membranes, neighboring signaling proteins, and lipid rafts, respectively (see Appendix for details).

In this study, we perform $5 \times 10^{7}$ attempted local moves per membrane lattice and simulate membranes with a size up to $100 \times 100$ lattices and periodic boundary conditions. The effective bending rigidity $\kappa$ of the membrane has a typical value of $12.5 k_{B} T$ [32] with $k_{B}$ being the Boltzmann constant and $T$ being the absolute temperature. In our model, the default values of TCR and pMHC concentrations are, respectively, 200 and 60 molecules $/ \mu \mathrm{m}^{2}$, which are consistent with experimental conditions [32,33]. These signaling proteins are randomly distributed through the nonraft regions of the membranes at the beginning of the simulations. According to Ref. [34], the complex length $l_{c}$ of TCR-pMHC complexes is set to be $15 \mathrm{~nm}$. Unless otherwise specified, we fix the depth of the square-well potential $U_{\text {binding }}=5.0 k_{B} T$ and potential range $l_{v}=1 \mathrm{~nm}$ [35] to define the TCR-pMHC interactions.

\section{RESULTS AND DISCUSSIONS}

\section{A. Effects of lipid raft characteristics on the TCR-pMHC interactions}

On the whole, the presence of lipid rafts will lead to a change in the overall diffusion rate of proteins on the membranes and an increase in local density of proteins in raft or nonraft regions. To facilitate the analysis, the static raft model is used here to uncover the underlying mechanism of the effect of lipid rafts on the TCR-pMHC interactions. 
(a)

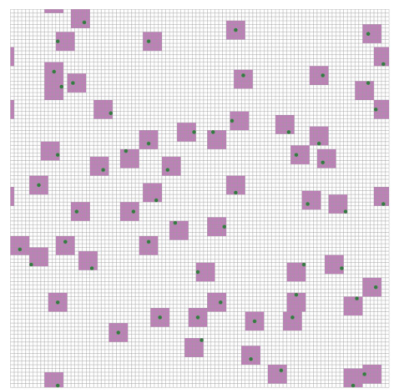

- pMHC $\square$ Raft in APC membrane

(b)

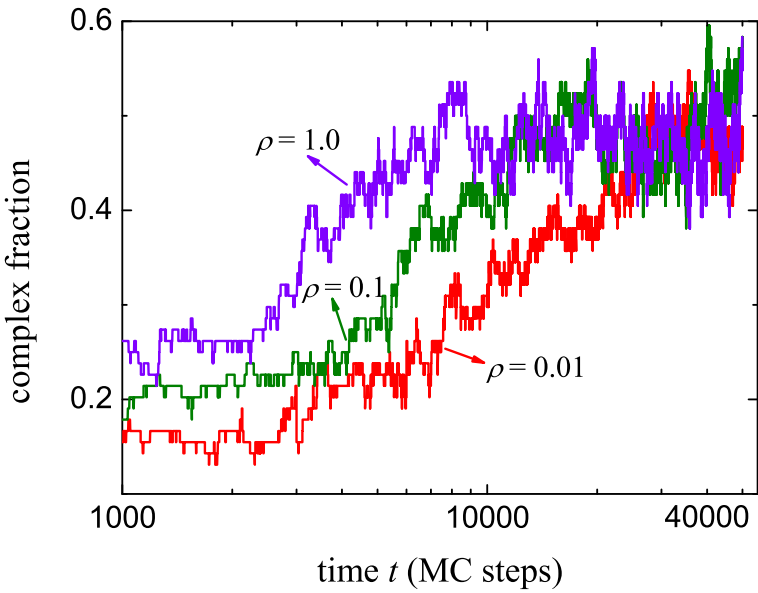

FIG. 2. (a) Snapshots of the spatial distribution of signaling proteins and lipid rafts in the T-cell and APC membranes at equilibrium. (b) Time evolution of TCR-pMHC complex fraction for three values of $\rho$. The number of permanently captured proteins in each lipid raft is 1 . Each lipid raft here has a fixed area of $50 \times 50 \mathrm{~nm}^{2}$. The parameter values used in the simulation are shown in Table I.

We first investigate the role of the diffusion rate of signaling proteins in the TCR-pMHC interactions by changing the diffusion ratio $\rho$. To rule out the influence of raft-induced protein aggregation, each lipid raft is allowed to carry only one signaling protein. At the beginning of the MC simulations, each raft is set to have high inherent affinity, $\Delta U_{\text {affinity }} \rightarrow-\infty$, for signaling proteins. However, if the chosen raft has already contained a protein during the simulation run, the raft affinity of this microdomain for proteins outside this area becomes $\Delta U_{\text {affinity }} \rightarrow+\infty$. Meanwhile, the number of lipid rafts is the same as that of signaling proteins in each membrane. As a result, at equilibrium the number of signaling proteins in each raft $N_{\text {pro }}$ will be the same and equal to 1 [Fig. 2(a)]. As shown in Fig. 2(b), although the time needed to reach equilibrium increases with smaller diffusion ratio $\rho$, all systems with different $\rho$ converge to the same level of TCR-pMHC complex fraction in the long run. Throughout this study, the complex fraction is defined as the ratio between the numbers of TCR-pMHC complexes and pMHCs. Consistently, the data shown in Fig. 3 indicate that the protein area concentration in lipid rafts and the TCR-pMHC complex fraction at equilibrium are independent of the parameters $\rho$ and $\phi$. Here, $\phi$ refers to the ratio of the diffusion coefficients of lipid rafts and signaling proteins. These results, which are in conformity with the standard thermodynamics theory, reveal that the diffusion rate of signaling proteins has no impact on the complex fraction at equilibrium. Moreover, this conclusion can be further verified by virtue of the existing theory. In the absence of lipid rafts, the membrane fraction $P_{b}$ within binding range for long receptor-ligand complexes has a linear relationship with rescaled effective potential depth $u$ and behaves as (Krobath et al. [35])

$$
P_{b} \approx c u=c a^{2}\left(\kappa / k_{B} T\right) l_{v}^{2}[R][L] e^{U / k_{B} T},
$$

with numerical prefactor $c \cong 13$ for small $P_{b} \leqslant 0.2$, where $[R]$ and $[L]$ are the area concentrations of unbound receptors and unbound ligands, respectively. Here, we obtain the dependence of $P_{b}$ on $u$ by varying the area concentration of signaling proteins in each membrane. For simplicity, the number of signaling protein (or lipid raft) in one membrane is set to be the same as that in the apposing membrane in Fig. 4. Meanwhile, the inherent affinity of a raft for protein molecules outside that ordered membrane domain undergoes a transition from $\Delta U_{\text {affinity }} \rightarrow-\infty$ to $\Delta U_{\text {affinity }} \rightarrow+\infty$ if the protein number inside that raft reaches a specified value $N_{\text {pro }}$. If each lipid raft is permitted to contain only one signaling protein, our results are in good agreement with the theoretical data in the absence of lipid rafts [Fig. 4(b)], which in turn demonstrates the validity of our model as well. It is important to note that although the raft diffusion coefficient has no effect on the complex fraction at equilibrium (Fig. 3), we use the Saffman-Delbruck equation [36] to calculate the size-dependent diffusion coefficient of lipid rafts and determine the difference in the diffusion frequency of lipid rafts and signaling proteins unless otherwise specified. This can ensure the rationality of our model in this respect (see Appendix for details).

Then we increase the number of signaling proteins in each lipid raft to investigate their accumulation effect within these microdomains on the intercellular bond formation. As described above, the raft affinity of $\Delta U_{\text {affinity }} \rightarrow-\infty$ becomes $\Delta U_{\text {affinity }} \rightarrow+\infty$ for signaling proteins outside this raft if the protein number inside that raft reaches a specified value $N_{\text {pro }}$, and the attempt for signaling proteins to enter into this raft will be rejected. The raft number in each membrane is set to be the protein number divided by this specified value $N_{\text {pro }}$. Consequently, at equilibrium each raft will carry the same number of signaling proteins [Fig. 4(a)]. It is clearly seen from Fig. 4(b) that the more the signaling proteins in each lipid raft, the higher the membrane fraction $P_{b}$ within binding range. These results indicate that the protein accumulation within lipid rafts facilitates the intercellular bond formation. Meanwhile, compared with the case of the absence of lipid rafts, a nonlinear relationship between $P_{b}$ and $u$ is presented for $N_{\text {pro }}>1$. By increasing the number of signaling proteins, it will result in the convergence phenomenon for large values of $u$, which is especially apparent for small values of $N_{\text {pro }}$, as shown in Fig. 4(b).

Finally, to investigate the role of lipid rafts in the intercellular TCR-pMHC interactions when the signaling proteins are excluded from these microdomains, we perform subsequent simulations with the raft affinity of $\Delta U_{\text {affinity }} \rightarrow+\infty$ [Fig. 5(a)]. The two apposing membranes contain the same number of lipid rafts, which is determined by raft area and size. Considering that lipid rafts are practically larger in size than single proteins, it can be concluded from Fig. 5(b) that the 
TABLE I. Simulation parameters for each figure. $n_{\text {component }}$ denotes the number of membrane components, including signaling proteins and lipid rafts in the T-cell membrane (T) and APC membrane (A). $\rho$ is the ratio of diffusion coefficients of signaling proteins inside and outside lipid rafts. $L_{\text {raft }}(\mathrm{nm})$ is the side length of the square raft in the static raft model. $U_{\alpha \alpha}$ is the attractive $c i s$ interactions of neighboring elementary units of lipid rafts in the dynamic raft model and $\left(U_{\alpha \alpha}\right)_{c}$ is the critical raft-raft interaction strength for the occurrence of phase separation. $N_{\text {pro }}$ is the protein number that each raft can carry. $A_{\text {raft }}$ is the raft area in the membranes. In Figs. 7-9, according to the experimental results, 50\% of total TCR molecules are set to reside in raft regions at equilibrium. The attempt to enter into a raft for TCRs in nonraft regions will be rejected when the specified number of TCRs inside the total raft regions is reached. In Figs. 7, 9, 11, and 12, to prevent the lipid rafts being filled up for high raft affinity $\Delta U_{\text {affinity }} \rightarrow-\infty$, the pMHC molecules in nonraft regions are no longer allowed to enter into these microdomains when they occupy $20 \%$ of the total raft regions.

\begin{tabular}{|c|c|c|c|c|c|c|c|c|c|}
\hline \multirow[b]{3}{*}{ Figure number } & \multicolumn{9}{|c|}{ Parameter } \\
\hline & \multirow[b]{2}{*}{$n_{\mathrm{TCR}}$} & \multirow[b]{2}{*}{$n_{\mathrm{pMHC}}$} & \multirow[b]{2}{*}{$\rho$} & \multicolumn{4}{|c|}{ Static raft model } & \multicolumn{2}{|c|}{ Dynamic raft model } \\
\hline & & & & $\overline{L_{\text {raft(T) }}}$ & $n_{\text {raft(T) }}$ & $L_{\text {raft(A) }}$ & $n_{\text {raft(A) }}$ & $U_{\alpha \alpha(\mathrm{T})}$ & $U_{\alpha \alpha(\mathrm{A})}$ \\
\hline Fig. 2 & 200 & 60 & $0.01-1.0$ & 50 & 200 & 50 & 60 & \multirow{15}{*}{$1.7\left(U_{\alpha \alpha}\right)_{c}$} & \\
\hline Fig. 3 & 200 & 60 & $0.01-1.0$ & 50 & 120 & 50 & 40 & & \\
\hline Fig. 4 & $50-350$ & $50-350$ & 0.01 & 50 & $n_{\mathrm{TCR}} / N_{\text {pro }}$ & 50 & $n_{\mathrm{pMHC}} / N_{\text {pro }}$ & & \\
\hline Fig. 5 & & & 0.01 & $10-90$ & $A_{\mathrm{raft}(\mathrm{T})} / L_{\mathrm{raft}(\mathrm{T})}^{2}$ & $10-90$ & $A_{\text {raft(A) }} / L_{\text {raft(A) }}^{2}$ & & \\
\hline \multirow{2}{*}{ Fig. 6} & & & 0.01 & 50 & 40 & 50 & 12 & & \\
\hline & & & & 50 & 300 & 50 & 300 & & \\
\hline Fig. 7 & & & 0.01 & 50 & 120 & $10-90$ & $A_{\text {raft(A) }} / L_{\text {raft(A) }}^{2}$ & & \\
\hline Fig. 8 & & & 0.01 & 50 & 120 & $10-180$ & $A_{\mathrm{raft}(\mathrm{A})} / L_{\mathrm{raft}(\mathrm{A})}^{2}$ & & \\
\hline Fig. 9 & 200 & 60 & 0.01 & & & & & & $0-2.0\left(U_{\alpha \alpha}\right)_{c}$ \\
\hline Fig. 10 & & & 0.01 & 50 & 120 & $10-90$ & $A_{\mathrm{raft}(\mathrm{A})} / L_{\mathrm{raft}(\mathrm{A})}^{2}$ & & \\
\hline Fig. 11 & & & 0.01 & & & $10-90$ & $A_{\mathrm{raft}(\mathrm{A})} / L_{\mathrm{raft}(\mathrm{A})}^{2}$ & & $0-2.0\left(U_{\alpha \alpha}\right)_{c}$ \\
\hline \multirow{4}{*}{$\begin{array}{ll} & (\mathrm{a} \\
& \\
& (\mathrm{b} \\
& (\mathrm{c} \\
& (\mathrm{d}\end{array}$} & & & 0.01 & & & $10-90$ & $A_{\text {raft(A) }} / L_{\text {raft(A) }}^{2}$ & & \\
\hline & & & 0.01 & & & & & & $0-2.0\left(U_{\alpha \alpha}\right)_{c}$ \\
\hline & & & 0.01 & & & 10-90 & $A_{\mathrm{raft}(\mathrm{A})} / L_{\mathrm{raft}(\mathrm{A})}^{2}$ & & \\
\hline & $200-3000$ & 60 & 0.01 & & & $10-90$ & $A_{\text {raft(A) }} / L_{\text {raft(A) }}^{2}$ & & \\
\hline
\end{tabular}

exclusion of proteins from lipid rafts promotes the intercellular TCR-pMHC interactions. This result can be understood as follows. Considering a situation that the raft coverage area is fixed at $50 \%$ of the membrane, the local protein concentration in nonraft regions is doubled when the raft affinity is $\Delta U_{\text {affinity }} \rightarrow$ $+\infty$. If the raft regions are occupied only by a single large raft, it will be easier for signaling proteins to aggregate in nonraft regions due to the poor size homogeneity, which favors in turn the complex formation. The size homogeneity can be improved if the single raft is divided into small ones. As a result, the

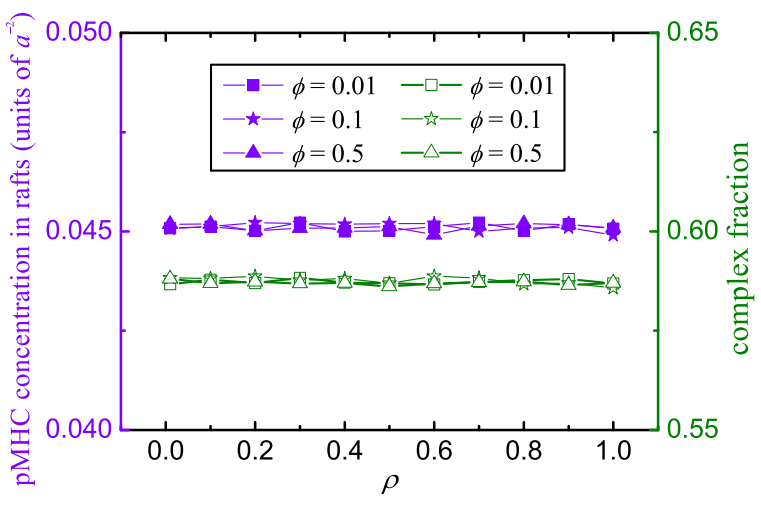

FIG. 3. Area concentration of pMHC molecules in lipid rafts and TCR-pMHC complex fraction as a function of the parameters $\rho$ and $\phi$ for raft affinity of $\Delta U_{\text {affinity }}=-3 k_{B} T$. The parameter values used in the simulation are shown in Table I. protein aggregation in nonraft regions will be weakened and vanish when the uniform size of lipid rafts is the same as that of signaling proteins. To quantitatively describe the aggregation behavior of signaling proteins in nonraft regions, the pair distribution function is defined as

$$
g(\mathbf{r})=\frac{1}{n_{\text {pro }}} \sum_{i=1}^{n_{\text {pro }}} g_{i}(\mathbf{r})=\frac{1}{n_{\text {pro }}} \sum_{i=1}^{n_{\text {pro }}} \frac{N_{i}(\mathbf{r})}{2 \pi \mathbf{r} \rho_{\text {pro }}} .
$$

Here, $n_{\text {pro }}$ and $\rho_{\text {pro }}$ denote the total number and area concentration of signaling proteins in each membrane, respectively. The subscript "pro" can be either TCR or pMHC. $N_{i}(\mathbf{r})$ is the protein number at a given distance $\mathbf{r}$ from the reference protein $i$. The pair distribution function gives the probability of finding a pair of proteins separated by a distance r. A larger value of $g(\mathbf{r})$ means a higher probability of finding a pair of proteins with a distance r. Specifically, $g(\mathbf{r})=1$ stands for a random distribution of signaling proteins. As shown in Fig. 5(c), the TCRs follow a random distribution in the T-cell membrane for lipid rafts with side length of $10 \mathrm{~nm}$ since the pair distribution function $g(\mathbf{r})$ stays around the value of 1 . As the raft size increases, the value of $g(\mathbf{r})$ is larger than 1 at smaller values of $\mathbf{r}$. This result indicates that the proteins tend to aggregate into a cluster for larger rafts. These results are in conformity with the above analysis.

In essence, the reason for the positive impact of aggregation effect on the intercellular TCR-pMHC interactions lies in binding cooperativity resulting from thermal membrane fluctuations [35]. It reveals that the extent of such promotive 
(a)

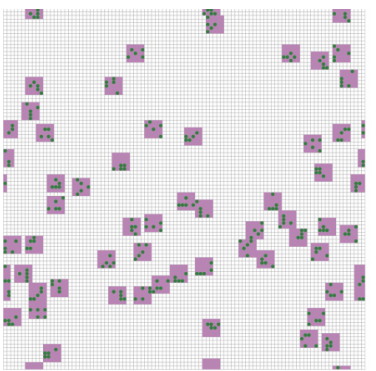

- pMHC $\square$ Raft in APC membrane

(b)

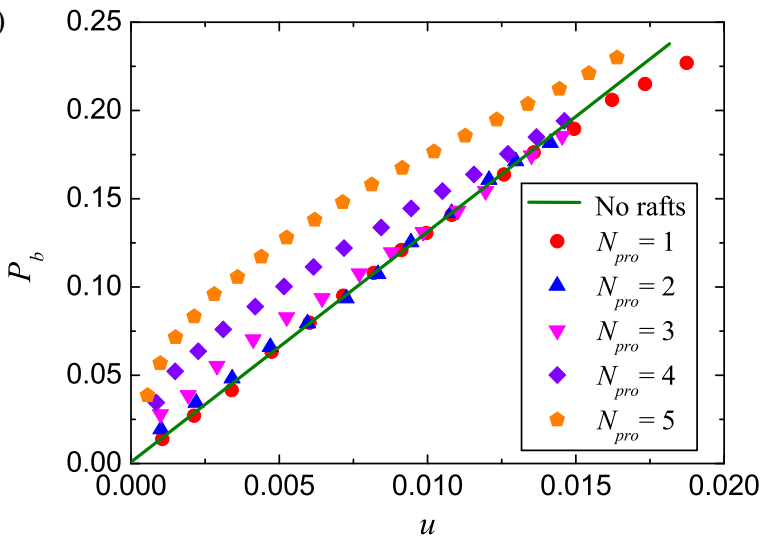

FIG. 4. (a) Snapshots of the equilibrium spatial distribution of signaling proteins and lipid rafts for protein number in each raft equaling 5. (b) Membrane fraction $P_{b}$ within binding range as a function of rescaled effective potential depth $u$ for lipid rafts loaded with different numbers of signaling proteins denoted by $N_{\text {pro }}$. Here, the dependence of $P_{b}$ on $u$ is obtained by varying the number of signaling proteins in each membrane. For simplicity, the number of TCRs is set to be the same as that of pMHC in that case. The raft number is determined by the ratio of protein number and $N_{\text {pro }}$. The parameter values used in the simulation are shown in Table I.

effect weakens with increasing $\kappa$, and specifically, the positive impact completely vanishes in the case of $\kappa \rightarrow \infty$ due to the disappearance of membrane fluctuations (Fig. 6).

\section{B. Lipid rafts can enhance the $2 \mathrm{D}$ in situ TCR-pMHC interactions}

In the following, a complete set of simulations based on static and dynamic raft models will be performed to explore the effect of lipid rafts on the interactions of membrane-anchored TCR and pMHC molecules. According to the experimental observations, lipid rafts are modeled to represent $30 \%$ of the $\mathrm{T}$ cell membrane; $50 \%$ of total TCRs are set to reside in raft regions at equilibrium [4,22,37]. Depending on the detection methods, the lipid rafts in the T-cell membrane vary widely in size. Here, we select a more recent consensus value of $50 \mathrm{~nm}$ to define the dimension of lipid rafts in the T-cell membrane [13,25,38-41]. Adopting the approach used in experiments [27], we investigate the impact of lipid rafts on the TCR-pMHC interactions by changing the characteristics of lipid rafts on APCs. The number of proteins in a lipid raft depends on the packing density, and thus, the protein number in a raft may statistically scale with the raft size. But there exists an upper limit for the protein number in a raft. For example, a (a) Raft size:

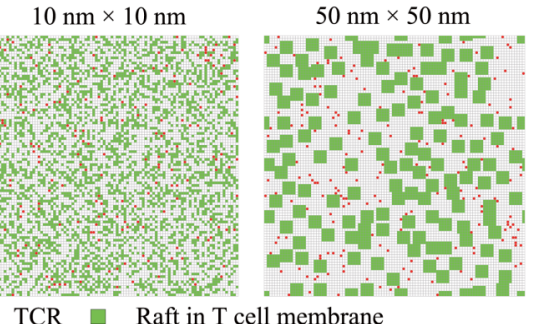

$90 \mathrm{~nm} \times 90 \mathrm{~nm}$

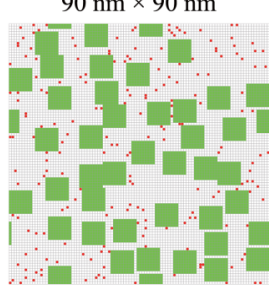

- TCR $\square$ Raft in T cell membrane

(b)

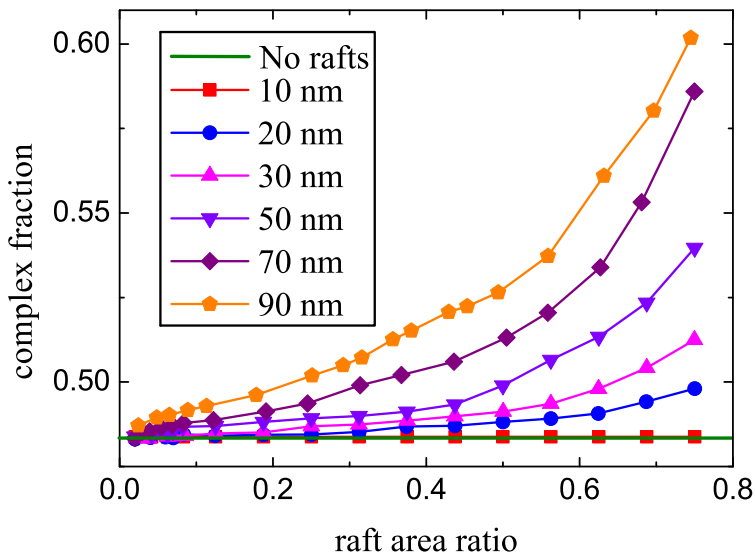

(c)

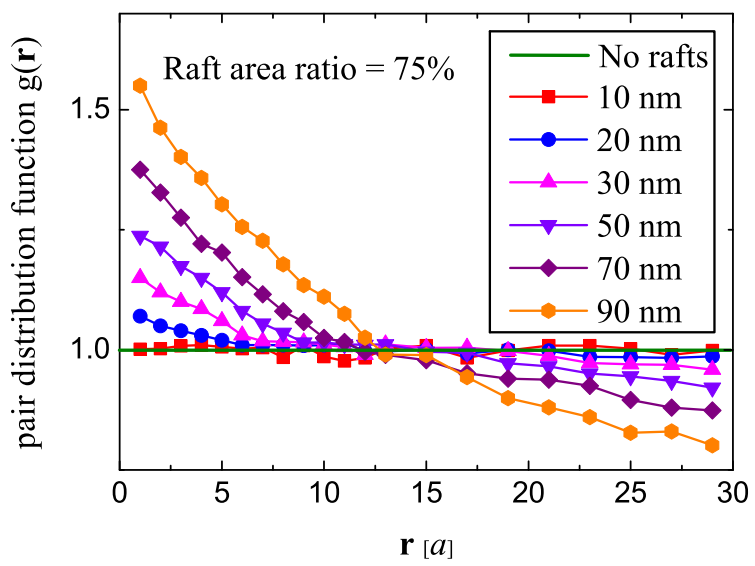

FIG. 5. (a) Snapshots of the equilibrium spatial distribution of TCRs and lipid rafts for raft affinity $\Delta U_{\text {affinity }} \rightarrow+\infty$ and raft making up $35 \%$ of the T-cell membrane with different side lengths. (b) Effect of the exclusion of signaling protein from lipid rafts on the complex fraction for different raft sizes. (c) Pair distribution function $g(\mathbf{r})$ as a function of the distance $\mathbf{r}$ for raft making up $75 \%$ of the membranes with raft side lengths ranging from 10 to $90 \mathrm{~nm}$. The raft size and area are set to be the same in T-cell and APC membranes in this figure. The parameter values used in the simulation are shown in Table I.

raft $50 \mathrm{~nm}$ in diameter would probably carry no more than 10-30 proteins $[4,42,43]$. However, the lipid rafts may be packed with signaling proteins for the extremely high raft affinity in our model (i.e., $\Delta U_{\text {affinity }} \rightarrow-\infty$, discussed below). To prevent this, we need to specify a constraint condition. In our static raft model, the lipid raft with size identical to a single protein molecule will be filled up once a signaling protein hops into this small microdomain. If we set a threshold value of $0.2 / a^{2}$ as the critical protein area concentration for a static raft, the lipid rafts with fixed sizes of $a \times a$ and 


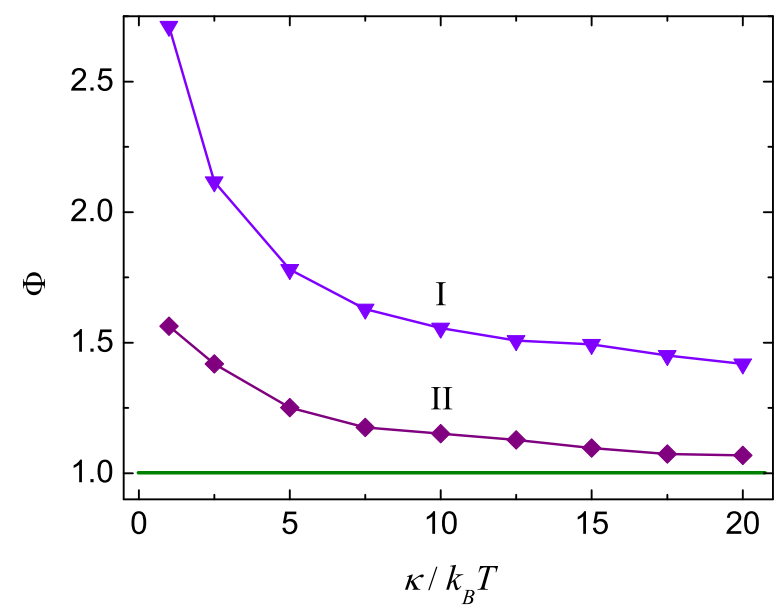

FIG. 6. Influence of thermal membrane fluctuations on the positive contribution made by the aggregation effect of signaling proteins inside and outside lipid rafts. $\Phi$ is the ratio of the number of TCR-pMHC complexes in the presence and absence of lipid rafts. In case I, the equilibrium protein number in each raft is 5 . In case II, lipid rafts make up $75 \%$ of the T-cell and APC membranes with raft affinity $\Delta U_{\text {affinity }} \rightarrow+\infty$. The side length of the lipid rafts is $50 \mathrm{~nm}$ in the two cases. The parameter values used in the simulation are shown in Table I.

$2 a \times 2 a$ will fail to carry any signaling proteins. Considering these actual situations, we define the finite protein-carrying capability in terms of the protein area concentration in the total raft coverage areas, rather than in an individual raft. Here, the signaling proteins account for no more than $20 \%$ of total raft coverage areas for high raft affinity $\Delta U_{\text {affinity }} \rightarrow-\infty$. This constraint applies to the dynamic raft model as well. We set the parameter $\Delta U_{\text {affinity }} \rightarrow-\infty$ to characterize the raft affinity for proteins at the beginning of the simulation. The raft affinity for proteins in nonraft regions becomes $\Delta U_{\text {affinity }} \rightarrow+\infty$ if the specified protein number in the total raft regions or the protein-carrying capability of the total raft regions is reached. In other words, the attempt to enter into a raft for TCRs in nonraft regions will be rejected when the number of TCRs inside the total raft regions is 100 , and the rest of the pMHCs outside lipid rafts are no longer allowed to enter into these microdomains when their area concentration in the total raft regions is $0.2 / a^{2}$ [Fig. $\left.7(\mathrm{~b})\right]$.

Figure 7(a) shows our simulation result from the static raft model that indicates a complicated interplay of lipid raft characteristics on the intercellular TCR-pMHC interactions. Taking into account the physical truth, we focus only on the results for lipid rafts that are larger than signaling proteins. We can clearly see that the TCR-pMHC complex fraction is promoted to different extents as a function of the lipid raft coverage and size. More specifically, the complex fraction exhibits a striking biphasic relationship with raft coverage. The number of TCR-pMHC complexes reaches its maximum value when lipid rafts on APC exactly accommodate all pMHCs. Meantime, we find that the correlation between the raft size and the complex fraction is basically positive for lipid rafts with size in the range of $20-90 \mathrm{~nm}$.

These results shown in Fig. 7(a) are definitely attributed to the affected distribution of signaling proteins in cell

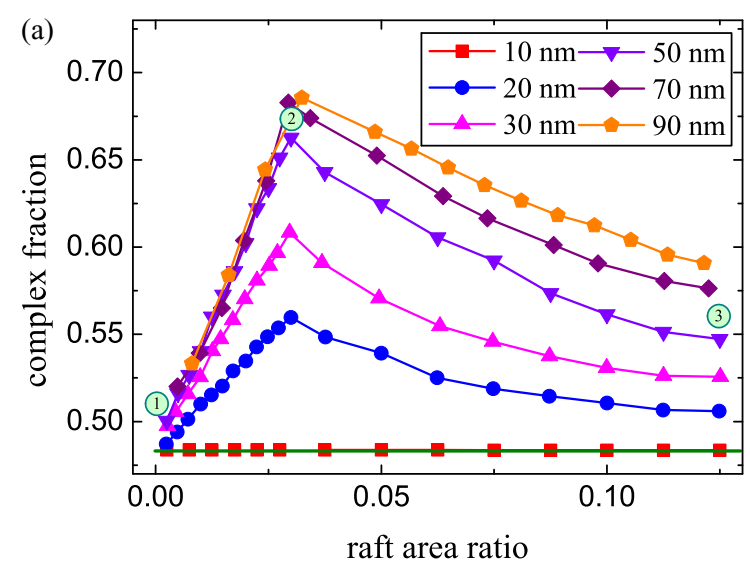

(b) (1)
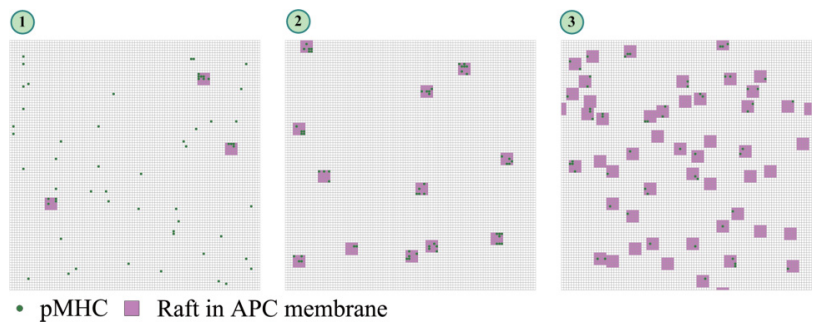

- pMHC $\square$ Raft in APC membrane

(c) 2

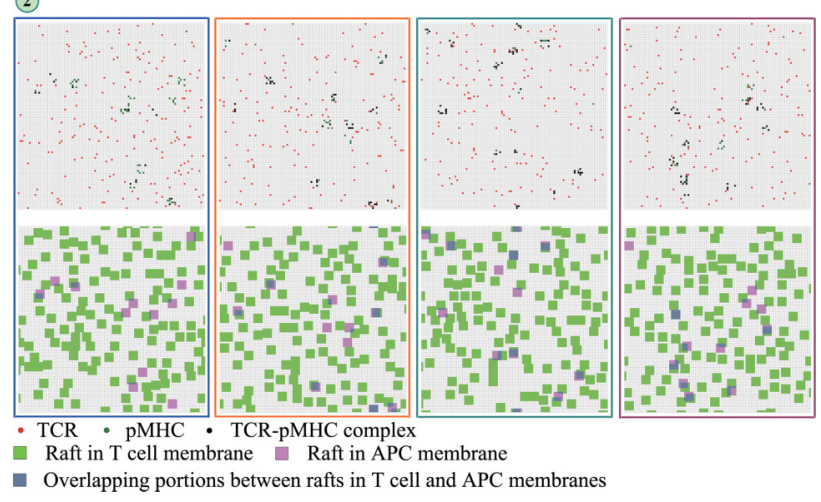

FIG. 7. (a) TCR-pMHC complex fraction as a function of the raft area ratio and raft size with parameters derived from experimental measurements. The olive line shows the values obtained in the absence of lipid rafts in the APC membrane. (b) Snapshots of the equilibrium spatial distribution of pMHCs and lipid rafts for three different raft areas marked with the numbers (1), (2), (3) corresponding to those in (a). The raft affinity is set as $\Delta U_{\text {affinity }} \rightarrow-\infty$ at the beginning of simulations. Here, the pMHCs are set to occupy at most $20 \%$ of the total raft regions due to the finite protein-carrying capability. The raft affinity for pMHCs in nonraft regions becomes $\Delta U_{\text {affinity }} \rightarrow+\infty$, and the pMHCs will not be allowed to enter into these microdomains if the protein-carrying capability of the total raft regions is reached. As a result, the equilibrium area concentration of pMHC in the total raft regions has fixed at $0.2 / a^{2}$ before the raft area is increased to a value large enough to be capable of exactly adsorbing all the pMHC molecules (1) $\rightarrow$ (2)). However, with further increase in raft area, the equilibrium area concentration of pMHC within the total raft regions decreases gradually (2) $\rightarrow$ (3)). (c) Time sequence of spatial distribution of signaling proteins and lipid rafts (2), top view). The lipid rafts carrying TCRs on T cell at equilibrium incline to be located in the membrane apposing the lipid rafts carrying pMHCs on APC. The snapshots are taken at $10^{3}, 10^{5}, 10^{6}$, and $10^{7} \mathrm{MC}$ steps. The final snapshot represents an equilibrium state. 
membranes due to the presence of lipid rafts. According to the analysis in Sec. III A, the aggregation of signaling proteins within lipid rafts can promote the formation of TCR-pMHC complexes, whereas the resulting concentration decrease of signaling proteins outside lipid rafts directly impairs the bond formation in nonraft regions. The increased concentration of TCR-pMHC complexes in the presence of lipid rafts indicates that the net contribution made by the competing effects is positive. With the increase in raft coverage for fixed raft size, the positive net contribution to TCR-pMHC interactions is enhanced, leading to the positive correlation between complex fraction and raft area ratio for small raft coverage. After the peak value of complex fraction is reached when all pMHCs reside in lipid rafts, there will be a decrease in the concentration of pMHCs in lipid rafts if further increasing raft area [Fig. 7(b)]. The reduced concentration directly impairs the aggregation effect of pMHCs inside lipid rafts, and in turn results in a negative correlation between complex fraction and raft area ratio for large raft coverage. Similarly, the change in raft size will also induce competing effects on the complex formation. On the one hand, decreasing the raft size for fixed raft coverage can facilitate the TCR-pMHC interactions by reducing the membrane roughness with more dispersive anchor points to clamp the two membranes together; on the other hand, it can impose a detrimental effect on the positive contribution made by aggregation effect inside lipid rafts. These factors compete and determine the raft-size-associated behaviors. The results from Fig. 7(a) show that, in most cases, the TCR-pMHC complex fraction increases with the raft size ( $\leqslant 90 \mathrm{~nm} \times 90 \mathrm{~nm}$ ), implying the positive effects of increasing raft size hold advantage. Additionally, according to the results in Sec. III A, the intercellular TCR-pMHC interactions are promoted when the signaling proteins are excluded from lipid rafts and tend to aggregate in nonraft regions. This positive contribution can also be affected by the raft area and size. However, note that the raft occupying area in the APC membrane used in Fig. 7(a) is relatively low $(<15 \%)$; thus the related effects on the complex formation can be negligible [see Fig. 5(b)].

To determine what extent the lipid rafts can contribute to the TCR-pMHC complex formation under physiologically relevant conditions, we further perform simulations for lipid rafts with larger size using the static raft model, considering the experimental result that more than half of the total amount of pMHCs is present in lipid rafts [27,44]. Figure 8(a) shows that the TCR-pMHC complex fraction increases with the size of lipid rafts on APC ( $\leq 180 \mathrm{~nm})$ and the amount of pMHCs in lipid rafts. Quantitatively, the binding affinity

$$
K_{2 \mathrm{D}}=\frac{[R L]}{[R][L]}
$$

that can be used to describe the strength of TCR-pMHC interactions, is enhanced by, on average, two- to threefold for $50 \%-100 \%$ of cell surface pMHCs residing in lipid rafts in view of the typical raft size of 100-200 nm in diameter on APCs [45-47]. Here, $[R L]$ indicates the area concentration of bound complexes. Moreover, it can even induce more than a fivefold increase in the TCR-pMHC binding affinity for all pMHCs residing in the lipid rafts with side length of $180 \mathrm{~nm}$ if we simultaneously consider the contribution of the lipid

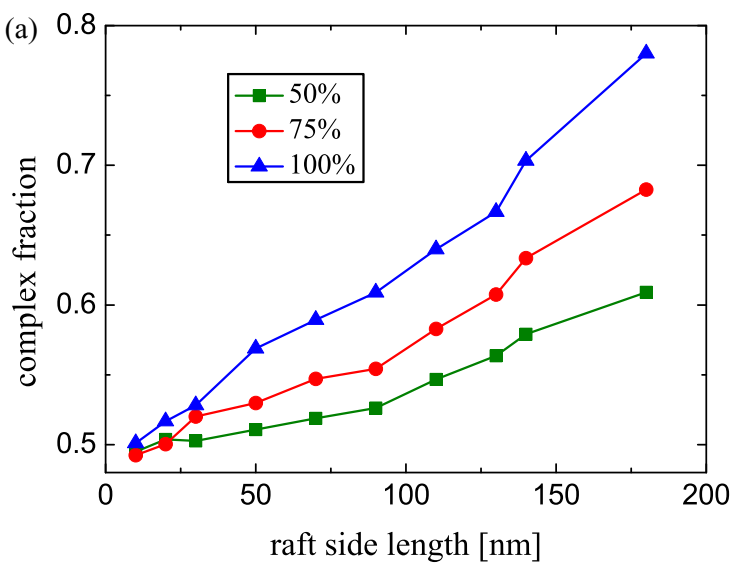

(b) Percentage of pMHCs in lipid rafts

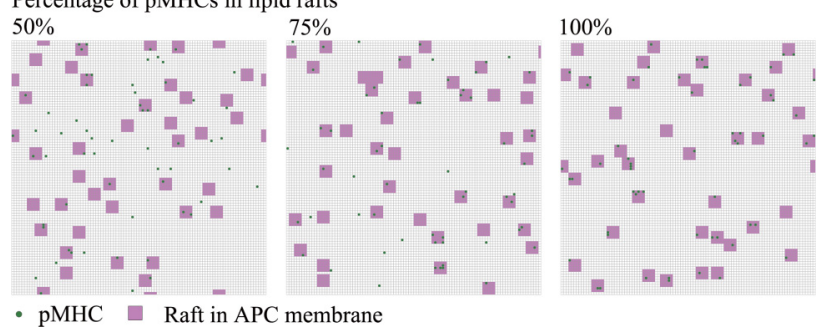

FIG. 8. (a) The extent to which lipid rafts facilitate the TCRpMHC bond formation as a function of raft size for different amounts of pMHC in lipid rafts. According to the experimental results, lipid rafts cover $10 \%$ of the APC surface [51]. The parameter values used in the simulation are shown in Table I. (b) Snapshots of the equilibrium spatial distribution of pMHCs and lipid rafts for three concentrations of pMHCs in these microdomains. The pMHC molecules in nonraft regions are no longer allowed to enter into lipid rafts when the specified pMHC-carrying capability of the total raft regions is reached.

rafts in the T-cell membrane. It has been well known that, as a prominent feature of the TCR signal initiation machinery, T-cell activation can be triggered by specific TCR recognition of the very low density of pMHCs in physiological conditions, but the underlying molecular mechanisms remain unclear. To date, various models for T-cell antigen recognition at such low density of pMHCs have been proposed, some of which have highlighted the roles of the coreceptor assistance [48] and CD3 conformational change [49]. Serial engagement of a single pMHC with clustered TCRs is also considered [50]. However, in these previous works, the lipid raft-based regulation has not been taken into account. Here, our results have borne out the significant promotive effect of lipid rafts on the TCR-pMHC binding affinity, which further extends the sustained interaction time crucially required for a full activation of a $\mathrm{T}$ cell. Our analysis might provide an alternative insight into the T-cell antigen receptor triggering mechanisms.

We next investigate the role of lipid rafts in the intercellular TCR-pMHC interactions using a dynamic raft model, in which lipid rafts undergo dynamic rearrangement such as merging and separating events modulated by the attractive $c i s$ interaction $U_{\alpha \alpha}$ they experience. Figure 9 (a) shows the effect of dynamic rafts on the formation of TCR-pMHC complex. The attractive interaction between neighboring elementary units of lipid rafts on a T-cell membrane is set as $U_{\alpha \alpha}=1.7\left(U_{\alpha \alpha}\right)_{c}$, 


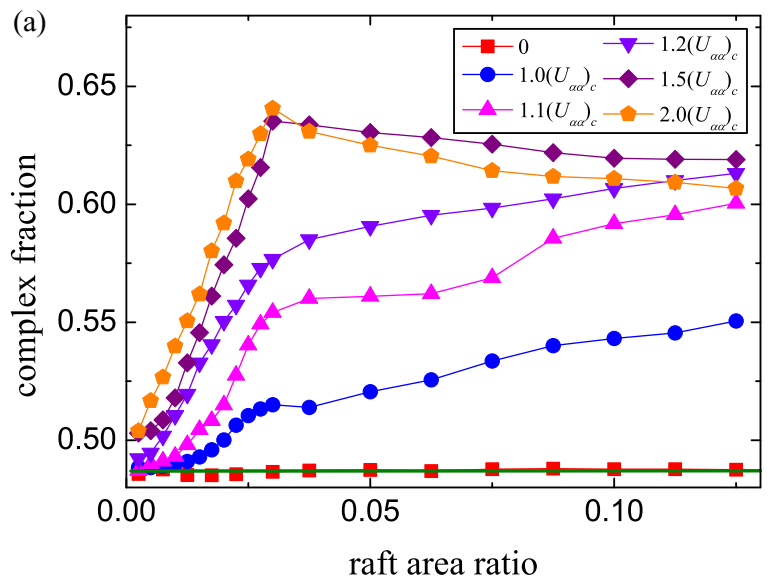

(b) Raft area ratio $=0.125$
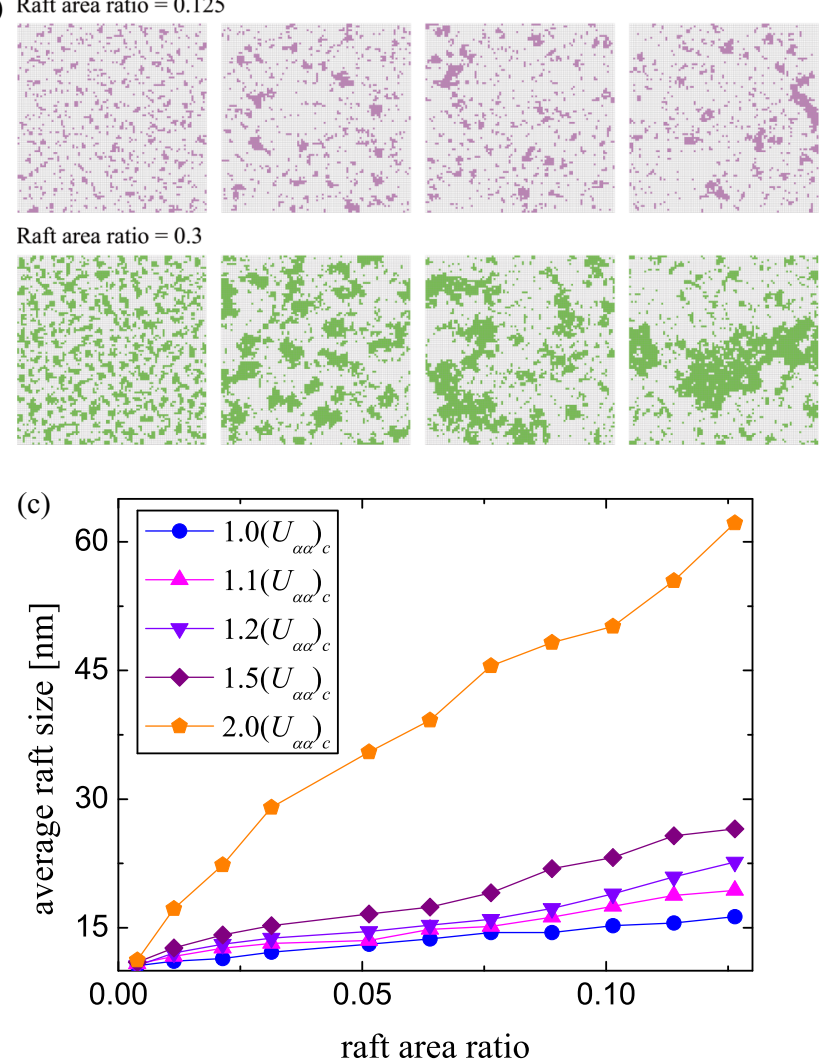

FIG. 9. (a) TCR-pMHC complex fraction as a function of the raft area ratio under five different attractive interaction values $U_{\alpha \alpha}$. The olive line shows the values obtained in the absence of lipid rafts in the APC membrane. (b) Time sequence of spatial distribution of dynamic rafts experiencing attractive interaction $U_{\alpha \alpha}=\left(U_{\alpha \alpha}\right)_{c}$ for different raft coverage areas. The snapshots are taken at $10^{3}, 10^{5}, 10^{6}$, and $5 \times 10^{7} \mathrm{MC}$ steps. The final snapshots represent the equilibrium state. (c) The average size of lipid rafts in the APC membrane as a function of the raft area ratio under five different attractive interaction values $U_{\alpha \alpha}$.

where $\left(U_{\alpha \alpha}\right)_{c}$ is the critical value of attractive interaction $U_{\alpha \alpha}$ for the occurrence of phase separation. Then we define the average equivalent side length of lipid rafts as $\bar{L}_{\text {raft(A) }}=$ $\sum \psi_{j} L_{j} / \sum \psi_{j}$, which is about $50 \mathrm{~nm}$ at equilibrium for the attractive interaction $U_{\alpha \alpha}=1.7\left(U_{\alpha \alpha}\right)_{c}$ and the raft coverage area of $30 \%$. Here, $\psi_{j}$ refers to the number of lipid rafts with side length of $L_{j}$. For the range of parameter values used here, the average size of lipid rafts on the APC membrane increases with raft coverage area or attractive interaction $U_{\alpha \alpha}$; see Fig. 9(c). As shown in Fig. 9(a), the TCR-pMHC complex fraction is promoted for dynamic rafts with average size larger than that of proteins due to the presence of attractive interaction $U_{\alpha \alpha}$. In accordance with the results in Fig. 7(a), the complex fraction increases with the average raft size characterized by raft area ratio and $U_{\alpha \alpha}$ before the critical raft area required for exactly accommodating all pMHCs is reached [Fig. 9(a)]. The trend of complex fraction with further increase in the raft area shows either a positive or negative correlation, which depends on the competition between the increased average raft size and the decreased area concentration of pMHC molecules in raft regions. A similar trend can be deduced from the data in Fig. 7(a). Quantitatively, there is a difference in the complex fraction obtained from the static and dynamic raft models, even though the raft size in the static raft model is the same as the average equivalent side length of lipid rafts in the dynamic raft model. This may be attributed to the dynamic changes in the size and shape of lipid rafts in the dynamic model.

Subsequently, in order to comprehensively investigate the effect of lipid rafts on the TCR-pMHC interactions, we consider a case that the lipid raft has a raft affinity of $\Delta U_{\text {affinity }}=-3 k_{B} T$. There is no longer constraint on the protein area concentration in the total raft coverage areas imposed in the case of $\Delta U_{\text {affinity }} \rightarrow-\infty$. The diffusion ratio $r=0.01$ [52] is used to describe the difference in diffusivity of signaling proteins inside and outside lipid rafts. The simulation results from the static raft model reveal that the TCR-pMHC complex fraction increases biphasically with the raft area. Meanwhile, the promotive effect strongly depends on the raft size ranging from 20 to $90 \mathrm{~nm}$ [Fig. 10(a)]. These results are mainly attributed to the multiple, competing effects as discussed in the case of Fig. 7(a). Additionally, comparing the results in Fig. 10(a) with those in Fig. 7(a), we find that the increasing intervals of complex fraction slightly increase with the raft side lengths in the range of $20-90 \mathrm{~nm}$. This may be caused by the different area concentrations of pMHC molecules in lipid rafts with different sizes. Our simulation results, as expected, show that the area concentration of pMHC molecules inside the total raft regions for different raft coverage areas is independent of the raft size in the absence of TCR-pMHC interactions. However, it reveals that the area concentration of $\mathrm{pMHC}$ molecules within raft regions increases with the raft side lengths ranging from 20 to $90 \mathrm{~nm}$ in the presence of TCR-pMHC interactions. This is different from the case in Fig. 7(a). In comparison with the smaller rafts, the positive contribution made by the protein aggregation in larger rafts can be more dominant when competing with the negative contributors discussed in Sec. III B due to the higher protein concentration within them. Then the increasing interval of complex fraction for larger rafts is extended.

\section{Lateral mobility of proteins on the substrate affects the role of lipid rafts in the complex formation}

The complexity of biological membranes and their interactions with the surrounding media make it challenging to directly investigate the cell-cell interactions. For this reason, 


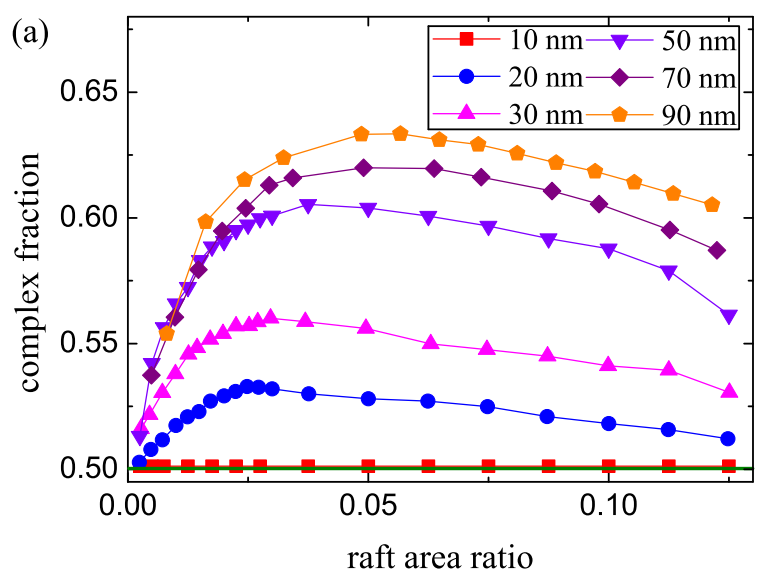

(b)

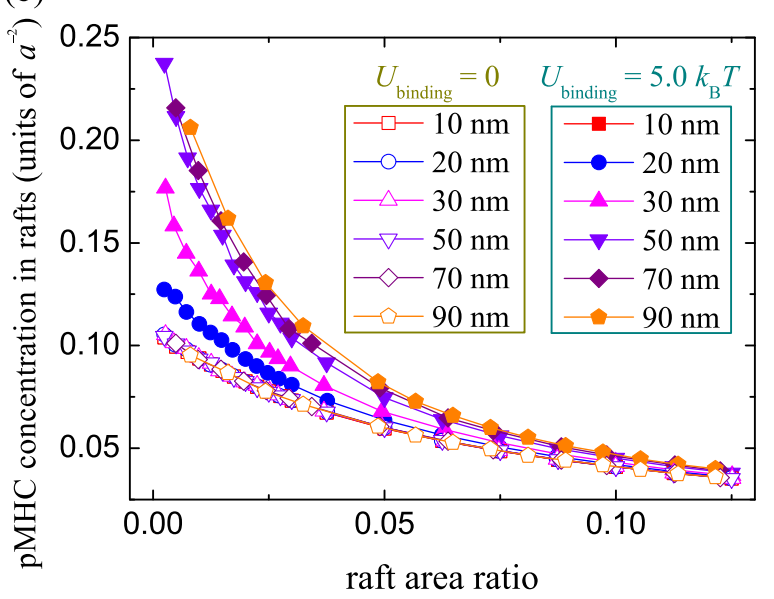

FIG. 10. (a) TCR-pMHC complex fraction as a function of the raft area ratio and raft size for raft affinity of $\Delta U_{\text {affinity }}=-3 k_{B} T$. (b) The concentration of pMHC molecules in raft regions as a function of the raft area ratio and size in the absence $\left(U_{\text {binding }}=0\right)$ and presence $\left(U_{\text {binding }}=5.0 k_{B} T\right)$ of TCR-pMHC interactions. The parameter values used in the simulation are shown in Table I.

a wide variety of biomimetic model systems have been developed. Among them, the 2D cell-substrate system has been commonly used in different experimental techniques and has provided significant new insights into the TCR recognition of pMHC in immune responses [53].

The cell-substrate model is typically constituted of a flat protein-containing lipid bilayer supported on a solid surface such as mica, glass, or silicon. The lipids and proteins within the supported membranes retain their lateral mobility which is a crucial characteristic for these studies [54]. To probe the issue concerned in this work, we adopt an experimental strategy that APCs interact with TCRs' functionalized supported membranes, or vice versa. Importantly, the implications of a costimulatory molecule on the TCR-pMHC interactions can be eliminated comparing with the in situ measurement for live $\mathrm{T}$ cells and APCs. This advantage, along with the tractability and controllability of a solid-supported membrane model, makes it possible to obtain more quantitative information on the behaviors of the TCR-pMHC interactions in the presence of lipid rafts. As shown in Fig. 11, the combined effects of both increased effective bending rigidity and the lipid rafts
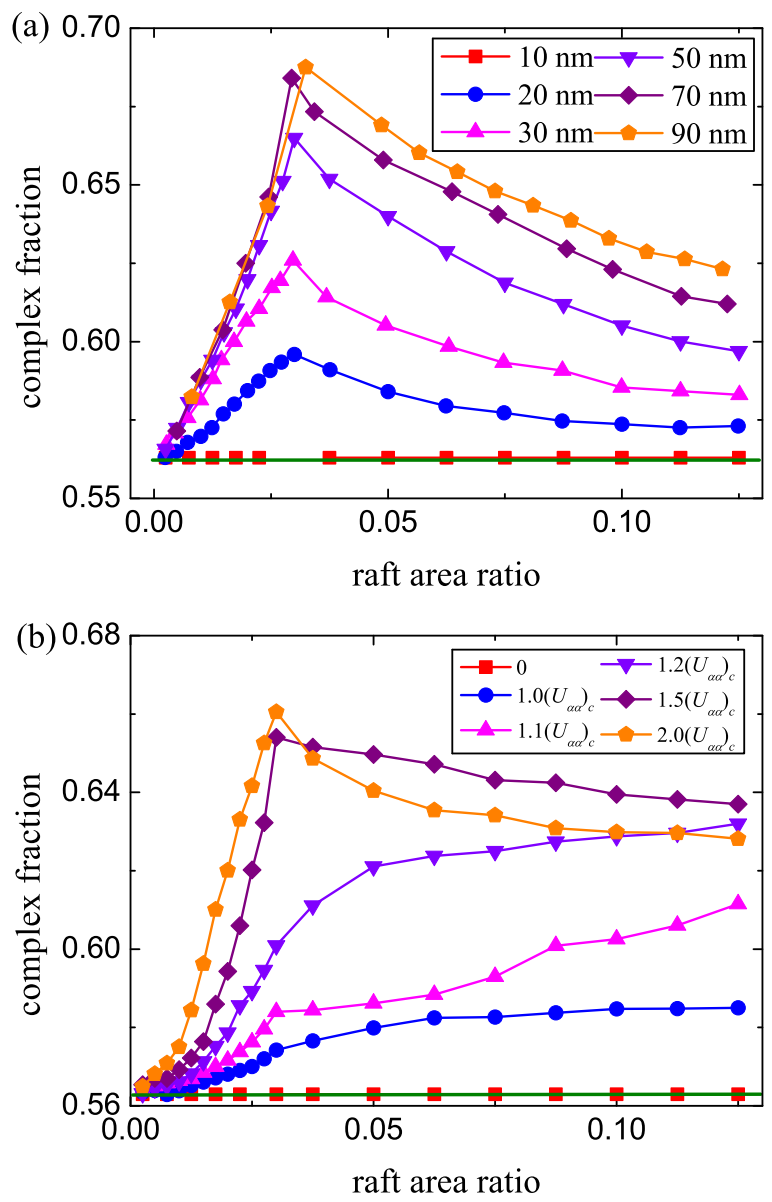

FIG. 11. The influence of lipid rafts on the TCR-pMHC interaction for the cell-substrate model constituted of a flat TCR-containing lipid bilayer supported on a solid substrate. The simulation results in $(\mathrm{a}, \mathrm{b})$ are from the static and dynamic raft models, respectively. The olive line shows the values obtained in the absence of lipid rafts in the APC membrane. The parameter values used in the simulation are shown in Table I.

that disappeared in the solid-supported membrane do not lead to qualitative differences in the impact of lipid rafts on the TCR-pMHC ligation. These results may provide the basis for further experimental research.

Another commonly used cell-substrate model for probing receptor-ligand interactions consists of a substrate presenting immobilized proteins. Detailed information on the molecularbinding properties has been derived from this simplified model. Here, in order to study the impact of lipid rafts on the TCR-pMHC interactions, we perform the following simulations where the TCRs are fixed on the substrate. From Figs. 12(a) and 12(b), it is surprising to find that the lipid rafts adversely affect the formation of TCR-pMHC bonds. In comparison with the case of Fig. 11, the complex fraction exhibits an opposite trend with increasing raft coverage when the side length of the lipid raft is larger than $10 \mathrm{~nm}$. Meanwhile, it reveals that the negative effect strongly depends on the raft size. These intriguing phenomena could also be attributed to the aggregation effect of lipid rafts on the membrane-anchored proteins. Intuitively, the lateral mobility of pMHC molecules in lipid rafts will be restricted due to the aggregation effect, 

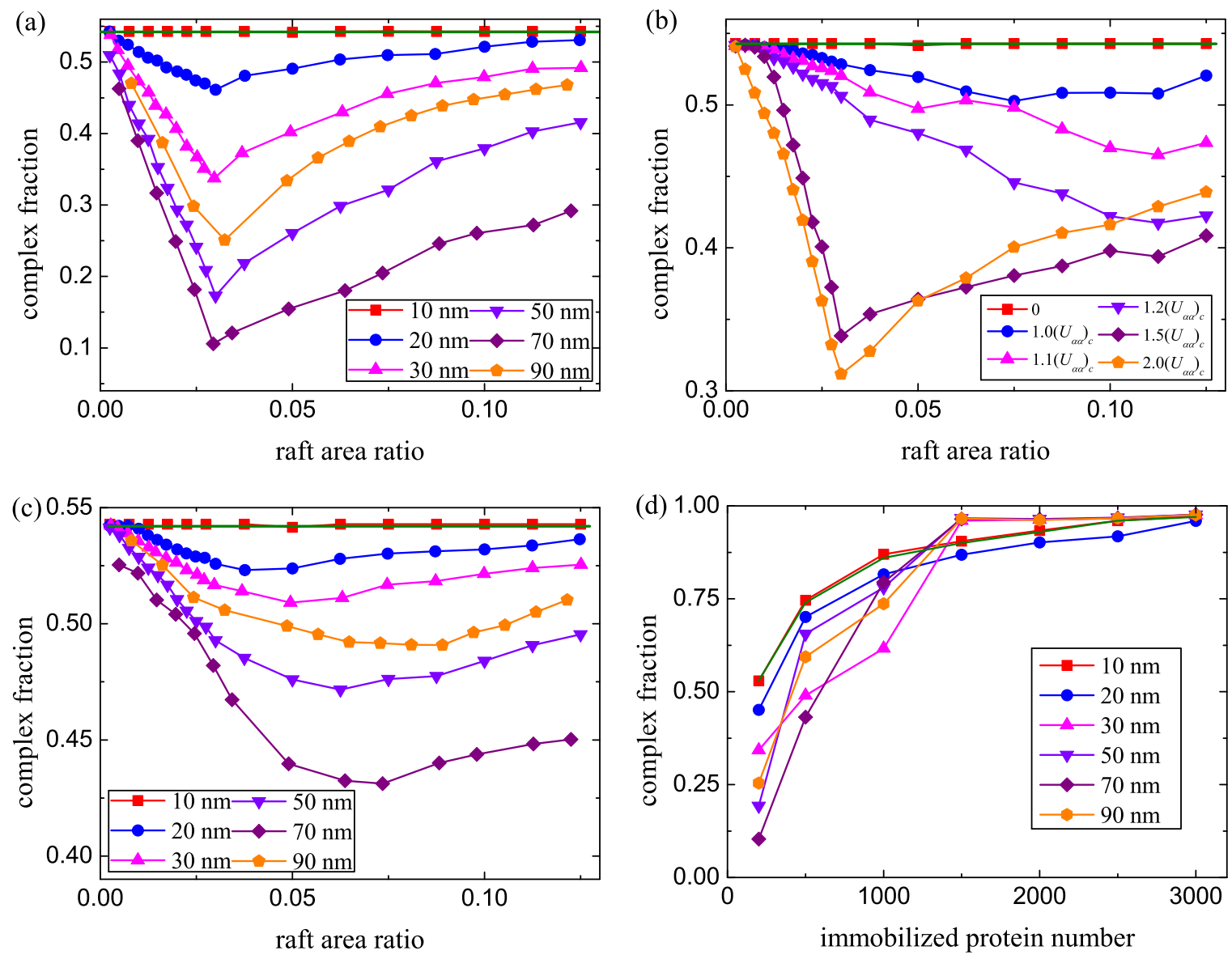

FIG. 12. Simulation results from the static (a) and dynamic (b) raft models showing the negative regulation of lipid rafts on the TCR-pMHC interactions for the case of immobilized TCRs coated on the substrate. As in Figs. 7(a) and 9(a), the signaling proteins can occupy no more than $20 \%$ of the total raft regions. (c) Detrimental effect of lipid rafts on the complex formation for cell-surface pMHCs and immobilized TCRs in the case where raft affinity $\Delta U_{\text {affinity }}=-3 k_{B} T$. (d) Complex fraction with respect to immobilized protein number and raft size for all pMHCs residing in these microdomains. The area concentration of pMHC molecules in the total raft regions is $0.2 / a^{2}$. The results in subfigures $(\mathrm{c}, \mathrm{d})$ are obtained from the static raft model. The olive line shows the values obtained in the absence of lipid rafts in the APC membrane. The parameter values used in these panels are shown in Table I.

which sterically interdicts their interactions with substrateimmobilized TCRs outside the sphere of influence of the lipid raft, thus preventing the bond formation. Likewise, the protein aggregation outside lipid rafts will give rise to unfavorable effects on the complex formation. The results presented in Figs. 12(a) and 12(b) originate predominantly from lipidraft-induced change in membrane protein distribution, and related effects have been discussed in Sec. III B. Consistently, as a result of aggregation effect, the presence of lipid rafts also shows a detrimental effect on the complex formation where signaling proteins have more realistic raft affinity [see Fig. 12(c)]. As expected, the inhibitory effect can be overcome by grafting the substrate with a high density of immobilized TCRs [Fig. 12(d)], because of the increased encounter and rebinding probabilities which weaken the negative impact of protein aggregation. Using this kind of cell-substrate model, Murai et al. [28] experimentally examined the case that $\mathrm{T}$ cells were perfused across hyaluronan-coated microtiter plates and conversely suggested that the presence of lipid rafts in the T-cell membrane suppresses cell adhesion and migration. Combined with the modeling results, we provide an explanation for this discrepancy regarding the role of lipid rafts in the receptor-ligand interactions. One should notice that, to investigate the effect of lipid rafts on the receptor-ligand interactions, Anderson et al. [27] and Murai et al. [28] used two different systems in which the interacting partners are TCR and pMHC, and CD44 and hyaluronan, respectively. This, however, may involve other potential underlying mechanisms accounting for the discrepancy. Alternatively, as speculated by Murai et al. [28], disruption of lipid rafts may trigger the conformational change that alters the hyaluronan-binding ability of CD44, which needs to be further studied.

In addition to the cell-substrate model, Murai et al. [28] also experimentally studied the effect of lipid rafts on the intermolecular interactions between membrane-anchored CD44 and in-solution hyaluronan. They showed that the hyaluronan-binding ability of CD44 in T cells is upregulated by raft disruption. Employing a stochastic MC algorithm, Caré and Soula [55] also found that the complex formation on the cell surface is inhibited by the presence of lipid rafts in this kind of receptor-ligand interaction. For binding of ligands diffusing in solution to the membrane-anchored 
receptors, there is no external force acting on the receptorligand complexes, whereas the 2D in situ receptor-ligand interaction will be negatively affected by the entropic repulsive force arising from thermally excited membrane fluctuations, as receptors and ligands anchor on two apposing surfaces. As discussed above, the presence of lipid rafts can lead to protein aggregation, which in turn promotes the $2 \mathrm{D}$ interactions essentially due to the fluctuation-induced cooperative binding of membrane-anchored receptors and ligands. Based on our current simulation results, we speculate that it may be the thermal membrane fluctuations that account for the contradictory results concerning the impact of lipid raft on the receptorligand interactions obtained by the two measurement methods.

Additionally, in the experiments of Anderson et al. [27] and Murai et al. [28], M $\beta \mathrm{CD}$ is used to extract the membrane cholesterol to investigate the role of lipid rafts in the receptor-ligand interactions. It is generally believed that $\mathrm{M} \beta \mathrm{CD}$-induced cholesterol depletion will lead to a decrease in the bending rigidity of the membranes [56-58], which in turn enhances the thermal fluctuations of the membranes. Thus the raft-disrupting agent, $\mathrm{M} \beta \mathrm{CD}$, plays a negative regulation role in the $2 \mathrm{D}$ receptor-ligand interactions from this perspective. However, more studies reveal that the effect of cholesterol depletion on the membrane bending rigidity is not universal but rather depends on the cell type and lipid architecture [59-62]. Therefore, the potential contributions of $\mathrm{M} \beta \mathrm{CD}$ to the receptor-ligand interactions may be specific to the type of cells and lipids.

\section{CONCLUSIONS}

Using a statistical-mechanical membrane model, we have investigated the impact of lipid rafts on the interactions between TCR and pMHC under different experimental conditions. We show that whether the contribution of lipid rafts to TCR-pMHC interactions is positive or negative depends strongly on the measurement methods. For the case of in situ molecular interactions where TCR and pMHC are anchored on the 2D membranes of the apposing cells, our results are consistent with the experimental data indicating that the presence of lipid rafts facilitates the intercellular TCR-pMHC interactions. We attribute the enhanced intercellular interactions to the lipid-raft-induced aggregation effect and thermal membrane fluctuations, which provides us with an alternative mechanism for lipid-raft-mediated regulation of intercellular signal transduction. Further quantitative analysis reveals a significant increase in the binding affinity of membrane-anchored TCR and $\mathrm{pMHC}$ under physiological conditions, which may provide an alternative insight into the mechanism of efficient T-cell activation at a very low density of pMHCs. In the cell-substrate models, our results indicate that the preservation of lateral mobility of signaling proteins on the solid substrate is indispensable for bringing into play the positive role of lipid rafts in facilitating TCR-pMHC interactions. Conversely, we find that, as a result of the immobilization of proteins on the substrate, protein aggregation induced by the presence of lipid rafts will function as a negative regulatory mechanism for the complex formation. These findings help to illuminate the seemingly contradictory experimental results. This study enriches our understanding of the functional implications of lipid rafts for the adaptive immune responses and suggests that we should prudently choose the experimental methods when measuring the impact of lipid rafts on the receptor-ligand interactions.

\section{ACKNOWLEDGMENTS}

L.L. thanks R. Lipowsky, T. R. Weikl, K. Simons, I. Parmryd, and J. L. Hu for insightful discussions. This work was supported by the Programs in the National Natural Science Foundation of China (Grants No. 11472285, No. 11232013, and No. 11402193), the Strategic Priority Research Program of the Chinese Academy of Sciences (Grant No. XDB22040102), and the National Key Research and Development Program of China (Grant No. 2016YFA0501601).

\section{APPENDIX: MODEL AND SIMULATION METHOD}

We use a statistical-mechanical membrane model [63] in which two membranes are interconnected by membraneanchored receptors (TCRs) and ligands (pMHCs). The lipid rafts can freely diffuse in the membranes.

\section{Discretized membrane model and TCR-pMHC interactions}

In the Monte Carlo (MC) simulation, we employ a discretized membrane model in which the spatial coordinates are replaced by a square grid with lattice site $i$ and lattice constant $a$, and the local separation describing the membrane conformations is specified by $l_{i}$. The membranes can collide, but cannot overlap, so that $l_{i} \geqslant 0$. The repulsive hard-wall interaction is implicitly embodied via the interaction potential,

$$
W_{\text {mem }}\left(l_{i}\right)=\infty \text { for } l_{i}<0 \text { and zero otherwise, }
$$

which induces the Helfrich entropic force [29,64]. The linear lattice size $a$ is set to be $10 \mathrm{~nm}$ to roughly follow the size of the TCR and pMHC [65]. Each lattice can contain a single signaling protein. Each TCR or pMHC in a certain lattice can hop by a distance of one lattice spacing to one of the four nearestneighbor lattices with equal probability during a single time step, provided that the available lattice is unoccupied by other molecules. The repulsive hardcore $c i s$ interaction between two neighboring TCRs or pMHCs at sites $i$ and $j$, which leads to their mutual exclusion within the membrane, has the form

$$
W_{\text {pro }}(i, j)=\infty \text { for } i=j \text { and zero otherwise. }
$$

In our model, whether a TCR-pMHC binding event occurs or not depends on the distribution of signaling proteins in each membrane patch and the local separation of binding sites [66]. The membrane configurations are then regulated by its bending rigidity and the specific TCR-pMHC interaction. Consequently, the effective Hamiltonian for the two fluctuating membranes interconnected by TCRs and pMHCs can be given by

$$
\begin{aligned}
H\{l, m, n\}= & H_{\mathrm{el}}\{l, m, n\}+H_{\text {int-pro }}\{l, m, n\} \\
& +W_{\text {mem }}\left(l_{i}\right)+W_{\text {pro }}(i, j) \\
= & \frac{\kappa}{2 a^{2}} \sum_{i}\left(\Delta_{\mathrm{d}} l_{i}\right)^{2}+\sum_{i} m_{i} n_{i} V\left(l_{i}\right) \\
& +W_{\text {mem }}\left(l_{i}\right)+W_{\text {pro }}(i, j),
\end{aligned}
$$


which is the essential element required for subsequent MC simulations. Here, $\kappa$ and $\Delta_{\mathrm{d}} l_{i}$ denote the effective bending rigidity and standard discretized Laplacian, respectively. $m_{i}$ $\left(n_{i}\right)$ describes the distribution of signaling proteins. If $m_{i}$ $\left(n_{i}\right)=1$, it means that there is a TCR (pMHC) in lattice $i$; otherwise, $m_{i}\left(n_{i}\right)=0$. We consider the square-well potential with the depth $U_{\text {binding }}>0$ and the range $l_{v}$ to characterize the TCR-pMHC interaction,

$$
V\left(l_{i}\right)=-U_{\text {binding }} \theta\left(l_{v} / 2-\left|l_{i}-l_{c}\right|\right),
$$

which is defined by a step function: $\theta(x)=1$ for $x \geqslant 0$, and $\theta(x)=0$ otherwise.

\section{Lipid raft model}

Lipid rafts are liquid-ordered microdomains with less fluidity that diffuse freely within the surrounding liquiddisordered membrane bilayer. Based on these properties associated with lipid rafts, we assign areas with low diffusivity as raft regions on the discretized membranes. The extent of the slowdown diffusion behavior of signaling proteins inside lipid rafts can be characterized by the diffusion coefficient ratio $\rho=D_{\text {raft }} / D_{\text {nonraft }}[30,31]$, where $D_{\text {raft }}$ and $D_{\text {nonraft }}$ represent the step size of signaling proteins inside and outside lipid rafts, respectively. Obviously, the smaller the ratio $\rho$, the more slowly the signaling proteins diffuse in lipid rafts. In our study, the unit step size for signaling proteins in raft and nonraft regions is set to be $\rho$ and one lattice, respectively. In our study, the lipid rafts are assumed to diffuse in a manner analogous to the proteins. Note that we use a Markov process to generate the random set of states in the MC simulations. An important condition we require for a Markov process is the condition of detailed balance. To satisfy this condition, the lipid rafts move independently of the signaling proteins in each membrane. This is somewhat different from the simulation process in Refs. [30,31] where the proteins residing in the lipid rafts move along with these microdomains. Similarly, to ensure volume exclusion and prevent overlaps of lipid rafts, repulsive hard-square interaction between microdomains is imposed, given by

$$
W_{\text {raft }}=\infty \text { for raft - raft overlaps and zero otherwise, }
$$

and the lateral diffusion of the lipid raft is only carried out when the target patches are not occupied by other rafts.

To reveal the impact of lipid rafts on the TCR-pMHC interaction, we employ two types-static and dynamicof lipid raft models. In the case of the static raft model, lipid rafts are modeled as square-shaped microdomains with defined sizes. The cis interaction between lipid rafts only includes the repulsive hard-square interaction, and there is no change in shape and size throughout the MC simulation. According to the Saffman-Delbrück equation [36], the ratio of the diffusion coefficients of microdomains $\left(D_{r}\right)$ and signaling proteins $\left(D_{p}\right)$, depending on their cross-sectional size, is given by

$$
\frac{D_{\mathrm{r}}}{D_{\mathrm{p}}}=\left(\ln \frac{\mu h}{\mu^{\prime} \bar{r}}-\gamma\right) /\left(\ln \frac{\mu h}{\mu^{\prime} r}-\gamma\right),
$$

where the viscosity of the fluid membrane $\mu=1$ poise, the viscosity of the surrounding aqueous phase $\mu^{\prime}=0.01$ poise, the Euler's constant $\gamma=0.5772$ [36], the thickness of the membranes $h$ and the radius of the signaling proteins $r$ are set to be $5 \mathrm{~nm}$ [67], and $\bar{r}$ denotes the equivalent radius of the square-shaped lipid rafts. For example, if $D_{r} / D_{p}=0.8$, a lipid raft has a probability of 0.8 to diffuse laterally and a probability of 0.2 not to move at all during a simulation step.

Lipid rafts in vivo are highly dynamic microdomains with a broad size distribution on the membranes. Additionally, lipid rafts undergo many merging and separating events during their lifetime [68]. Here, we present a dynamic raft model to mimic these processes, in which lipid rafts experience attractive $c i s$ interactions between them. Hu et al. [69] have adopted similar model to study the morphologies of thermally fluctuating vesicles. In general, the interaction energy of neighboring lattices for a single membrane is given by

$$
H_{\text {int-pat }}=\sum_{\langle i j\rangle} U_{i j},
$$

where $\langle i j\rangle$ denotes a nearest-neighbor pair of membrane patches and $U_{i j}$ is the corresponding interaction energy for each pair. Here, we use the notation $\alpha$ and $\beta$ to denote the raft and nonraft membrane patches, respectively. Each nearest-neighbor pair is one of the three types $(\alpha \alpha),(\beta \beta)$, or $(\alpha \beta)$. Letting the respective numbers of such pairs be $N_{\alpha \alpha}$, $N_{\beta \beta}, N_{\alpha \beta}$, Eq. (A7) becomes

$$
H_{\text {int-pat }}=N_{\alpha \alpha} U_{\alpha \alpha}+N_{\beta \beta} U_{\beta \beta}+N_{\alpha \beta} U_{\alpha \beta} .
$$

Considering the two-dimensional square-lattice geometry, we have the relations

$$
\begin{aligned}
& 4 N_{\alpha}=2 N_{\alpha \alpha}+N_{\alpha \beta}, \\
& 4 N_{\beta}=2 N_{\beta \beta}+N_{\alpha \beta},
\end{aligned}
$$

where $N_{\alpha}$ and $N_{\beta}$ are the total number of $\alpha$ and $\beta$ squares, respectively. According to Eq. (A9), the interaction energy of membrane patches in Eq. (A8) can be written as

$H_{\text {int-pat }}=N_{\alpha \beta}\left[U_{\alpha \beta}-\frac{1}{2}\left(U_{\alpha \alpha}+U_{\beta \beta}\right)\right]+2\left[N_{\alpha} U_{\alpha \alpha}+N_{\beta} U_{\beta \beta}\right]$,

where the last term on the right-hand side of the above equation is a constant. Then, the interaction energy $H_{\text {int-pat }}$ depends only on the value of $N_{\alpha \beta}$. Comparison of Eq. (A10) with the results from the Ising model without an external magnetic field immediately leads to the correspondence

$$
U_{\alpha \beta}-\frac{1}{2}\left(U_{\alpha \alpha}+U_{\beta \beta}\right)=2 J,
$$

where $J$ is the interaction energy between nearest-neighbor spins. With the exact critical value $J_{c} / k_{B} T=\ln (1+\sqrt{2}) / 2$ for lateral phase separation in the square-lattice Ising model [70], we get the relation

$$
\left[U_{\alpha \beta}-\left(U_{\alpha \alpha}+U_{\beta \beta}\right) / 2\right]_{c}=\ln (1+\sqrt{2}) k_{B} T .
$$

If $U_{\alpha \beta}=0$ and $U_{\beta \beta}=0$, one can obtain the critical raft-raft interaction strength,

$$
\left(U_{\alpha \alpha}\right)_{c}=-2 \ln (1+\sqrt{2}) k_{B} T .
$$

Equation (A13) provides an important reference value for modeling the phase behavior of lipid rafts. In the dynamic raft model, the elementary unit of lipid rafts is set to be a square lattice of size $a \times a$, and we modulate the dynamic properties 
of lipid rafts by varying $c i s$-interaction energy, $U_{\alpha \alpha}$, near its critical value, $\left(U_{\alpha \alpha}\right)_{c}$. The effective Hamiltonian of the system is then given by

$$
\begin{aligned}
H\{l, m, n\}= & \frac{\kappa}{2 a^{2}} \sum_{i}\left(\Delta_{\mathrm{d}} l_{i}\right)^{2}+\sum_{i} m_{i} n_{i} V\left(l_{i}\right)+W_{\text {mem }}\left(l_{i}\right) \\
& +W_{\text {pro }}(i, j)+W_{\text {raft }}-\frac{1}{2} N_{\alpha \beta} U_{\alpha \alpha}+2 N_{\alpha} U_{\alpha \alpha} .
\end{aligned}
$$

It should also be pointed out that, with regard to the formation of lipid rafts, there are a number of theoretical and computational models to mimic and elucidate the dynamic processes. Veatch et al. [71] suggested that compositional fluctuations at transient temperature are the origins of microdomain formation. Fan et al. [72] explained the formation of lipid rafts by studying the nonequilibrium lipid transport processes. Additionally, many studies emphasized the crucial roles of protein-lipid and protein-protein interactions in the formation and regulation of lipid rafts $[73,74]$. For more details on different models of raft formation, see Refs. [68,75]. These theories and models offer new insights for plasma membrane heterogeneity and provide alternative modeling perspectives for the dynamic simulations of lipid rafts.

One of the most important properties of lipid rafts is that they may include or exclude proteins. To simulate the raft affinity, we introduce a parameter $\Delta U_{\text {affinity }}$ to define the corresponding change in the energy for a signaling protein in nonraft regions entering into a lipid raft. Then the acceptance probability of the hopping process of the signaling proteins between nonraft and raft regions is determined by the Metropolis algorithm described below. In addition, the diffusion of a lipid raft may lead to a change in the energy by including or excluding signaling proteins. This process should also follow the standard Metropolis criterion. Particularly, once hopping into a lipid raft, a signaling protein will be permanently captured for $\Delta U_{\text {affinity }} \rightarrow-\infty$ through its hopping process.

In our model, the signaling proteins and lipid rafts are randomly sampled to undergo diffusion during each MC step. However, such sampling procedures will involve the unequal probability selection when there is a difference in the number of signaling proteins and lipid rafts, causing the TCRs and pMHCs, as well as lipid rafts, to fail to diffuse accurately according to the physical relations determined by the SaffmanDelbrück equation [36]. To address this problem, we can make use of the number ratios of signaling proteins and lipid rafts in the membranes of APC and T cell: $R_{\mathrm{TCR}}=n_{\mathrm{TCR}} / n_{\text {total }}$, $R_{\mathrm{pMHC}}=n_{\mathrm{pMHC}} / n_{\text {total }}, R_{\mathrm{A}}=n_{\text {raft(A) }} / n_{\text {total }}, R_{\mathrm{T}}=n_{\text {raft( } \mathrm{T})} / n_{\text {total }}$, together with a random number $R$ between 0 and 1. Only if the condition $R<R_{\text {component }}$ is satisfied can the corresponding component be chosen to attempt to move. Here, the subscript "component" can be either a signaling protein or a lipid raft in each membrane.

\section{Characteristic time scales associated with physical processes}

Importantly, we should take seriously the characteristic time scales associated with physical processes in our model. According to the two-dimensional diffusion law $t=a^{2} / 4 D$, the characteristic hop time of signaling proteins on the length scale $\alpha=10 \mathrm{~nm}$ is on the order of $2 \mathrm{~ms}$ with the typical diffusion constant $D \approx 10^{-10} \mathrm{~cm}^{2} / \mathrm{s}$ for membrane-anchored proteins [76], while the characteristic relaxation time for membrane shape fluctuations on this length scale is around $0.2 \mathrm{~ms}$ [77], tenfold smaller than that protein diffusion time. To handle this disparity in characteristic time scales, we perform simulations with, on average, ten attempted local moves per patch before all signaling proteins attempt to diffuse laterally once. Subsequently, each lipid raft will undergo the hopping process with a probability determined by the Saffman-Delbrück equation [36].

\section{Metropolis MC method}

In order to investigate the role of the lipid raft on the TCR-pMHC ligation, we employ standard Metropolis MC simulations for the model defined by Eq. (A14). During the simulations, both the fluctuations of membranes and the lateral diffusion of signaling proteins and lipid rafts may lead to a change in configurational energy of the system. Following the Metropolis criterion, the local fluctuation or the lateral diffusion is always accepted if the change in configurational energy $\Delta H$ is negative, but is accepted with the probability $\exp \left(-\Delta H / k_{B} T\right)$ for $\Delta H>0$.
[1] A. Prasada, J. Zikherman, J. Das, J. P. Roose, A. Weiss, and A. K. Chakraborty, Proc. Natl. Acad. Sci. U. S. A. 106, 528 (2009).

[2] J. Huang, V. I. Zarnitsyna, B. Y. Liu, L. J. Edwards, N. Jiang, B. D. Evavold, and C. Zhu, Nature 464, 932 (2010).

[3] L. Klein, B. Kyewski, P. M. Allen, and K. A. Hogquist, Nat. Rev. Immunol. 14, 377 (2014).

[4] K. Simons and D. Toomre, Nat. Rev. Mol. Cell Biol. 1, 31 (2000).

[5] H. P. Wang, T. A. Kadlecek, B. B. Au-Yeung, H. E. Goodfellow, L. Y. Hsu, T. S. Freedman, and A. Weiss, Cold Spring Harbor Perspect. Biol. 2, a002279 (2010).

[6] C. Klammt, L. Novotná, D. T. Li, M. Wolf, A. Blount, K. Zhang, J. R. Fitchett, and B. F. Lillemeier, Nat. Immunol. 16, 961 (2015).

[7] S. Yasukawa, Y. Miyazaki, C. Yoshii, M. Nakaya, N. Ozaki, S. Toda, E. Kuroda, K. Ishibashi, T. Yasuda, Y. Natsuaki,
F. Mi-ichi, E. Iizasa, T. Nakahara, M. Yamazaki, K. Kabashima, Y. Iwakura, T. Takai, T. Saito, T. Kurosaki, B. Malissen et al., Nat. Commun. 5, 3755 (2014).

[8] A. Mócsai, J. Ruland, and V. L. Tybulewicz, Nat. Rev. Immunol. 10, 387 (2010).

[9] M. Hinterberger, M. Aichinger, O. Prazeres da Costa, D. Voehringer, R. Hoffmann, and L. Klein, Nat. Immunol. 11, 512 (2010).

[10] G. L. Stritesky, S. C. Jameson, and K. A. Hogquist, Annu. Rev. Immunol. 30, 95 (2012).

[11] I. Kass, A. M. Buckle, and N. A. Borg, Trends Immunol. 35, 604 (2014).

[12] F. van Laethem, A. N. Tikhonova, L. A. Pobezinsky, X. Tai, M. Y. Kimura, C. Le Saout, T. I. Guinter, A. Adams, S. O. Sharrow, G. Bernhardt, L. Feigenbaum, and A. Singer, Cell 154, 1326 (2013). 
[13] M. Dykstra, A. Cherukuri, H. W. Sohn, S. J. Tzeng, and S. K Pierce, Annu. Rev. Immunol. 21, 457 (2003).

[14] S. Y. Na, A. Patra, Y. Scheuring, A. Marx, M. Tolaini, D. Kioussis, B. A. Hemmings, T. Hünig, and U. Bommhardt, J. Immunol. 171, 1285 (2003).

[15] S. Mahammad, J. Dinic, J. Adler, and I. Parmryd, Biochim. Biophys. Acta 1801, 625 (2010).

[16] B. F. Lillemeier, M. A. Mörtelmaier, M. B. Forstner, J. B. Huppa, J. T. Groves, and M. M. Davis, Nat. Immunol. 11, 90 (2010).

[17] J.-H. Cho, H.-O. Kim, C. D. Surh, and J. Sprent, Immunity 32, 214 (2010).

[18] P. A. van der Merwe and O. Dushek, Nat. Rev. Immunol. 11, 47 (2011).

[19] D. Filipp, O. Ballek, and J. Manning, Front. Immunol. 3, 155 (2012).

[20] M. Nagafuku, K. Okuyama, Y. Onimaru, A. Suzuki, Y. Odagiri, T. Yamashita, K. Iwasaki, M. Fujiwara, M. Takayanagi, I. Ohno, and J.-i. Inokuchi, Proc. Natl. Acad. Sci. U. S. A. 109, E336 (2012).

[21] H.-Y. Cheng, R. Wu, A. K. Gebre, R. N. Hanna, D. J. Smith, J. S. Parks, K. Ley, and C. C. Hedrick, PLoS One 8, e63746 (2013).

[22] J. Dinic, A. Riehl, J. Adler, and I. Parmryd, Sci. Rep. 5, 10082 (2015).

[23] A. K. Bamezai and W. Bakun, J. Immunol. 196, Suppl. 1, 128.1 (2016).

[24] D. Lingwood and K. Simons, Science 327, 46 (2010).

[25] K. Simons and M. J. Gerl, Nat. Rev. Mol. Cell Biol. 11, 688 (2010).

[26] H. T. He, A. Lellouch, and D. Marguet, Semin. Immunol. 17, 23 (2005).

[27] H. A. Anderson, E. M. Hiltbold, and P. A. Roche, Nat. Immunol. 1, 156 (2000).

[28] T. Murai, C. Sato, M. Sato, H. Nishiyama, M. Suga, K. Mio, and H. Kawashima, J. Cell Sci. 126, 3284 (2013).

[29] W. Helfrich, Z. Naturforsch. A 33, 305 (1978).

[30] D. V. Nicolau, Jr., K. Burrage, R. G. Parton, and J. F. Hancock, Mol. Cell. Biol. 26, 313 (2006).

[31] D. V. Nicolau, Jr., J. F. Hancock, and K. Burrage, Biophys. J. 92, 1975 (2007).

[32] T. R. Weikl and R. Lipowsky, Biophys. J. 87, 3665 (2004).

[33] A. Grakoui, S. K. Bromley, C. Sumen, M. M. Davis, A. S. Shaw, P. M. Allen, and M. L. Dustin, Science 285, 221 (1999).

[34] S. J. E. Lee, Y. Hori, and A. K. Chakraborty, Proc. Natl. Acad. Sci. U. S. A. 100, 4383 (2003).

[35] H. Krobath, B. Różycki, R. Lipowsky, and T. R. Weikl, Soft Matter 5, 3354 (2009).

[36] P. G. Saffman and M. Delbrück, Proc. Natl. Acad. Sci. U. S. A. 72, 3111 (1975).

[37] P. Drevot, C. Langlet, X. J. Guo, A. M. Bernard, O. Colard, J. P. Chauvin, R. Lasserre, and H. T. He, EMBO J. 21, 1899 (2002).

[38] C. Klammt and B. F. Lillemeier, Front. Immunol. 3, 291 (2012).

[39] T. Harder and K. R. Engelhardt, Traffic 5, 265 (2004).

[40] C. J. Fielding, Lipid Rafts and Caveolae: From Membrane Biophysics to Cell Biology (John Wiley \& Sons, Weinheim, 2006).

[41] C. Kennedy, M. D. Nelson, and A. K. Bamezai, Cell Commun. Signal. 9, 31 (2011).
[42] K. Simons and R. Ehehalt, J. Clin. Invest. 110, 597 (2002).

[43] M. Edidin, Annu. Rev. Biophys. Biomol. Struct. 32, 257 (2003).

[44] H. A. Anderson and P. A. Roche, Biochim. Biophys. Acta 1853, 775 (2015).

[45] M. B. Fessler and J. S. Parks, J. Immunol. 187, 1529 (2011).

[46] L. J. Pike, J. Lipid Res. 44, 655 (2003).

[47] K. Jacobson, O. G. Mouritsen, and R. G. W. Anderson, Nat. Cell Biol. 9, 7 (2007)

[48] N. R. J. Gascoigne, Nat. Rev. Immunol. 8, 895 (2008).

[49] W. W. A. Schamel, R. M. Risueno, S. Minguet, A. R. Ortiz, and B. Alarcon, Trends Immunol. 27, 176 (2006).

[50] J. Huang, C. Meyer, and C. Zhu, Mol. Immunol. 52, 155 (2012).

[51] H. T. He and D. Marguet, EMBO Rep. 9, 525 (2008).

[52] M. Fallahi-Sichani and J. J. Linderman, PLoS One 4, e6604 (2009).

[53] B. N. Manz, B. L. Jackson, R. S. Petit, M. L. Dustin, and J. Groves, Proc. Natl. Acad. Sci. U. S. A. 108, 9089 (2011).

[54] K. D. Mossman, G. Campi, J. T. Groves, and M. L. Dustin, Science 310, 1191 (2005).

[55] B. R. Caré and H. A. Soula, BMC Syst. Biol. 5, 48 (2011).

[56] I. Titushkin and M. Cho, Biophys. J. 90, 2582 (2006).

[57] G. Ng, K. Sharma, S. M. Ward, M. D. Desrosiers, L. A. Stephens, W. M. Schoel, T. Li, C. A. Lowell, C.C. Ling, M. W. Amrein, and Y. Shi, Immunity 29, 807 (2008).

[58] L. Amin, E. Ercolini, R. Shahapure, E. Migliorini, and V. Torre, Biophys. J. 102, 2451 (2012).

[59] F. J. Byfield, H. Aranda-Espinoza, V. G. Romanenko, G. H. Rothblat, and I. Levitan, Biophys. J. 87, 3336 (2004).

[60] J. Pan, S. Tristram-Nagle, and J. F. Nagle, Phys. Rev. E 80, 021931 (2009).

[61] N. Khatibzadeh, A. A. Spector, W. E. Brownell, and B. Anvari, PLoS One 8, e57147 (2013).

[62] R. Dimova, Adv. Colloid Interface Sci. 208, 225 (2014).

[63] G. K. Xu, J. L. Hu, R. Lipowsky, and T. R. Weikl, J. Chem. Phys. 143, 243136 (2015).

[64] L. Li and F. Song, Acta Mech. Sin. 32, 970 (2016).

[65] G. P. O’Donoghue, R. M. Pielak, A. A. Smoligovets, J. J. Lin, and J. T. Groves, eLife 2, e00778 (2013).

[66] T. R. Weikl, M. Asfaw, H. Krobath, B. Różycki, and R. Lipowsky, Soft Matter 5, 3213 (2009).

[67] D. Boal, Mechanics of the Cell (Cambridge University Press, Cambridge, 2012).

[68] J. Fan, M. Sammalkorpi, and M. Haataja, FEBS Lett. 584, 1678 (2010).

[69] J. L. Hu, T. Weikl, and R. Lipowsky, Soft Matter 7, 6092 (2011).

[70] W. Krauth, Statistical Mechanics: Algorithms and Computations (Oxford University Press, New York, 2006).

[71] S. L.Veatch, P. Cicuta, P. Sengupta, A. Honerkamp-Smith, D. Holowka, and B. Baird, ACS Chem. Biol. 3, 287 (2008).

[72] J. Fan, M. Sammalkorpi, and M. Haataja, Phys. Rev. Lett. 100, 178102 (2008).

[73] N. Destainville, Phys. Rev. E 77, 011905 (2008).

[74] A. D. Douglass and R. D. Vale, Cell 121, 937 (2005).

[75] J. Fan, M. Sammalkorpi, and M. Haataja, Phys. Rev. Lett. 104, 118101 (2010)

[76] A. J. Bean, Protein Trafficking in Neurons (Academic Press, San Diego, 2007).

[77] E. Lindahl and O. Edholm, Biophys. J. 79, 426 (2000). 